\title{
LA INTERVENCIÓN PSICOLÓGICA EN PACIENTES ONCOLÓGICOS: UNA REVISIÓN DE LA LITERATURA (2000-2014)
}

PSYCHOLOGICAL INTERVENTION IN ONCOLOGICAL PATIENTS: A REVIEW (2000-2014)

\author{
Erica Villoria' ${ }^{1}$ Concepción Fernández², Celina Padierna ${ }^{3}$ y Sonia González ${ }^{2}$ \\ ' Instituto de Estudios Sociales y Humanísticos. Universidad Autónoma de Chile, Chile \\ 2 Universidad de Oviedo. Oviedo (Asturias), España. \\ ${ }^{3}$ Servicio de Salud del Principado de Asturias (SESPA), España.
}

Resumen

Objetivo: Valorar la utilidad de las distintas intervenciones psicológicas en relación al estado emocional, físico, funcionalidad y la calidad de vida de los pacientes oncológicos en las diferentes fases de la enfermedad.

Método: Se realizó una búsqueda bibliográfica en las bases de datos Medline y CancerLit, entre el año 2000 y 2014.

Resultados: Un total de 122 estudios experimentales se describen agrupados según las fases de la enfermedad y los tipos de intervención psicológica aplicados. Se discute acerca de sus garantías metodológicas y se analizan las medidas y valoraciones de eficacia terapéutica para identificar las intervenciones con mejores pronósticos.

Conclusiones: En general, los trabajos revisados han logrado resultados favorables en relación a la mejora del estado emocional, físico, funcional y la calidad de vida de los pacientes. No obstante, sus limitaciones metodológicas (uso de muestras pequeñas, dificultades de generalización, ausencia de grupo control, etc.) $y$, en ocasiones, resultados contradictorios señalan cautela a la hora de concluir sobre la efectividad o no de la intervención psicológica en cáncer y los beneficios de su aplicación.

Palabras clave: Cáncer, intervención psicológica, estado emocional, síntomas físicos y calidad de vida.

\section{Abstract}

Objective: The aim of this work has been to assess the use of the different psychological interventions related to the emotional, physical, functional state and quality of life of cancer patients in the different phases of the illness.

Method: For this purpose, a bibliographic search between the years 2000 and 2014 in Medline and CancerLit databases was conducted.

Results: A total of 122 experimental studies are described grouped according to the illness phases and the kind of psychological intervention employed. It is discussed about the methodological guaranties and the measures and the assessments of therapeutic efficiency to identify the interventions with the best prognostic.

Conclusions: In general, the studies reviewed have achieved favorable results in relation to the improvement of the emotional, physical, functional state and the quality of life the patients. Nevertheless, their methodological limitations (little samples, difficulties of generalization, control group absence, etc.) and, sometimes, contradictory results suggest to be cautious when concluding about the effectiveness or not of the psychological intervention in cancer and the benefits of its application.

Keywords: Cancer, psychological intervention, emotional state, physical symptoms and quality of life.

\section{Correspondencia:}

Erica Villoria Fernández.

Instituto de Estudios Sociales y Humanísticos. Campus El Llano Subercaseux. Universidad Autónoma de Chile

Ricardo Morales N 3369, Comuna San Miguel, Ciudad Santiago de Chile. Código Postal: 8910132

E-mail: erica.villoria@uautonoma.cl 


\section{PLANTEAMIENTO DEL PROBLEMA}

La enfermedad oncológica implica síntomas y alteraciones asociadas con la enfermedad y/o tratamiento que ya desde el diagnóstico, pueden suponer limitaciones y malestar emocional con la consiguiente repercusión en la calidad de vida de los pacientes $^{(1-4)}$. La prevención y tratamiento psicológico de la población oncológica ha generado un amplio campo de investigación en las últimas tres décadas. Diferentes trabajos han obtenido resultados favorables, a pesar de utilizar diferentes criterios de evaluación y clasificación ${ }^{(5-7)}$. No obstante, Lepore y Coyne (2006) ponen de manifiesto claras diferencias entre los trabajos de revisión bibliométrica a la hora de concluir sobre la efectividad o no de la intervención psicológica en cáncer y los beneficios de su aplicación ${ }^{(8)}$.

A pesar de las posibles discrepancias entre los resultados, existe consenso en la necesidad de abordar todas las circunstancias que rodean al paciente y a su entorno (síntomas, limitaciones, alteraciones de las rutinas diarias, problemas emocionales, etc.), que, además, pueden ser distintas en función de cada fase o momento de la enfermedad y/o tratamiento. Estas fases por las que el paciente puede pasar en el transcurso de la enfermedad, junto al pronóstico de la misma, pueden resultar críticas para determinar los objetivos generales y el formato más adecuado de intervención ${ }^{(9)}$.

Ante la escasez de revisiones sobre la intervención psicológica en oncología en función de estas fases, el objetivo de este trabajo ha sido la revisión y exposición de los estudios que han analizado la utilidad de intervenciones psicológicas educativoinformativas, cognitivo-conductuales, psicoterapia individual y grupal en relación con el estado emocional, físico, funcionalidad y la calidad de vida de los pacientes oncológicos en cualquiera de las fases de la enfermedad.

\section{MÉTODO}

Se realizó una búsqueda exhaustiva en las bases de datos Medline, Pubmed y CancerLit, entre el año 2000 y 2014. Los descriptores utilizados para este fin fueron: "cancer and quality of life", "cancer and quality of life and review", "cancer and psychological intervention" $\mathrm{y}$ "cancer and psychological intervention and review". Además, con el objetivo de abarcar otros trabajos, incluso previos al año 2000, se han tenido en cuenta distintas revisiones publicadas por otros autores ${ }^{(5,7,9-20)}$.

La revisión se realizó partiendo de los artículos originales, escritos en inglés y español y de los resúmenes ofrecidos en las bases de datos, en los que se especificase el procedimiento y la muestra del estudio.

\section{RESULTADOS}

De los estudios revisados ( $\mathrm{N}=863)$ se seleccionaron 122 trabajos. Se incluyeron aquellos con pacientes de cáncer adultos a los que se aplicó una intervención psicológica para valorar su influencia en diferentes parámetros de salud (física, psicológica y social) y la calidad de vida. Se excluyeron aquellos trabajos en los que no se especificaba muestra, objetivos, procedimiento y resultados. Además, se tuvieron en cuenta los resultados ofrecidos por 15 revisiones publicadas por otros autores en relación con las intervenciones psicológicas aplicadas en cáncer. En atención a lo anterior, se presentan en las tablas sólo trabajos posteriores al año 2000.

En función de la fase de la enfermedad se encontraron, 28 artículos sobre la fase diagnóstica, 49 sobre la fase de tratamiento, 32 sobre la fase libre de enfermedad/ supervivencia y 12 sobre la fase de recidiva/enfermedad terminal. Se hallan diferentes porcentajes en relación con las intervenciones psicológicas empleadas 
por las diferentes investigaciones en cada una de las fases. En la fase de diagnóstico, el $39,3 \%$ corresponde a intervenciones psico-educativas, un $21,4 \%$ tanto para intervenciones cognitivo-conductuales como psicoterapéuticas individuales y un 17,4\% para grupales. En la fase de tratamiento el $24,5 \%$ fueron intervenciones psico-educativas, el $49 \%$ cognitivo-conductuales, un $16,3 \%$ psicoterapéuticas individuales y un $10 \%$ grupales. En la fase libre de enfermedad/ supervivencia, un $21,9 \%$ fueron intervenciones psico-educativas, un $34,4 \%$ cognitivo-conductuales, $28,1 \%$ psicoterapéuticas individuales y un $15,6 \%$ grupales. Por último, en la fase de recidiva/progresión y enfermedad terminal, un $16,7 \%$ fueron intervenciones psico-educativas, un $25 \%$ cognitivo-conductuales, $16,7 \%$ psicoterapéuticas individuales y un $41,7 \%$ grupales.

Con respecto a los objetivos perseguidos por las intervenciones psicológicas, se destacan los mayores porcentajes para la mejora de la calidad de vida $(28,7 \%)$, el estado emocional $(26,2 \%)$ y el control de síntomas $(13,9 \%)$.

Revisión de intervenciones psicológicas en la fase de sospecha y diagnóstico

Desde la perspectiva de proporcionar información y educación, las intervenciones se orientaron preferentemente a aspectos psicosociales como aspectos emocionales, apoyo y adaptación a la enfermedad. Mediante diferentes profesionales (enfermería especializada, psicólogos, trabajadores sociales, psiquiatras), la información fue proporcionada mediante folletos, libros o revistas, material audiovisual, charlas o reuniones; a través de internet o del teléfono, y mediante conversación tipo Counseling. Este tipo de intervenciones han mostrado efectos positivos en relación a mejorar la comunicación médico-paciente, mejorar el conocimien- to con respecto a la enfermedad y aspectos relacionados con los tratamientos y, ofrecer apoyo adicional para mejorar conocimientos sobre los recursos disponibles para la obtención de ayuda. Además, han logrado reducir el estrés, mejorar el estado afectivo (ansiedad y depresión) y el ajuste y afrontamiento a la enfermedad. Asimismo, algunos trabajos han apuntado su utilidad para mejorar la adherencia al tratamiento (véase Tabla 1). Estudios previos al año 2000 indican resultados en la misma dirección ${ }^{(21-25)}$.

Otros trabajos, en cambio, han señalado que aunque los pacientes bajo intervención psicológica obtienen mejoras, éstas no siempre resultan significativas en comparación con aquellos pacientes sin intervención ${ }^{(26-28)}$. Los trabajos que han aplicado paquetes cognitivo-conductuales (véase tabla 1), se han centrado en técnicas de solución de problemas, reestructuración cognitiva, imaginación guiada, biofeedback, relajación y entrenamiento en habilidades de afrontamiento. Éstos han sido utilizados por distintos profesionales de la salud, mostrándose adecuados para mejorar la ansiedad y respuesta inmunológica, favorecer la aceptación del diagnóstico, mejorar el ajuste y afrontamiento a la enfermedad y reducir el estrés ${ }^{(29,30)}$. Parecen idóneos para facilitar una alianza con el médico y el equipo de tratamiento y desarrollar e iniciar un plan de tratamiento. Desde los estudios de revisión, también se han señalado efectos positivos en relación con mejorar la calidad de vida del paciente ${ }^{(7,11-13,31-34)}$.

Las intervenciones psicoterapéuticas individuales en esta fase, según Fawcy et al. (1995), se orientaron fundamentalmente al establecimiento de una relación de empatía con el paciente con miras a disminuir el estrés y la ansiedad, proporcionar apoyo emocional y comprensión, y mejorar el ajuste y afrontamiento a la enferme$\operatorname{dad}^{(32,35,36)}$. En otros casos, se han centrado 


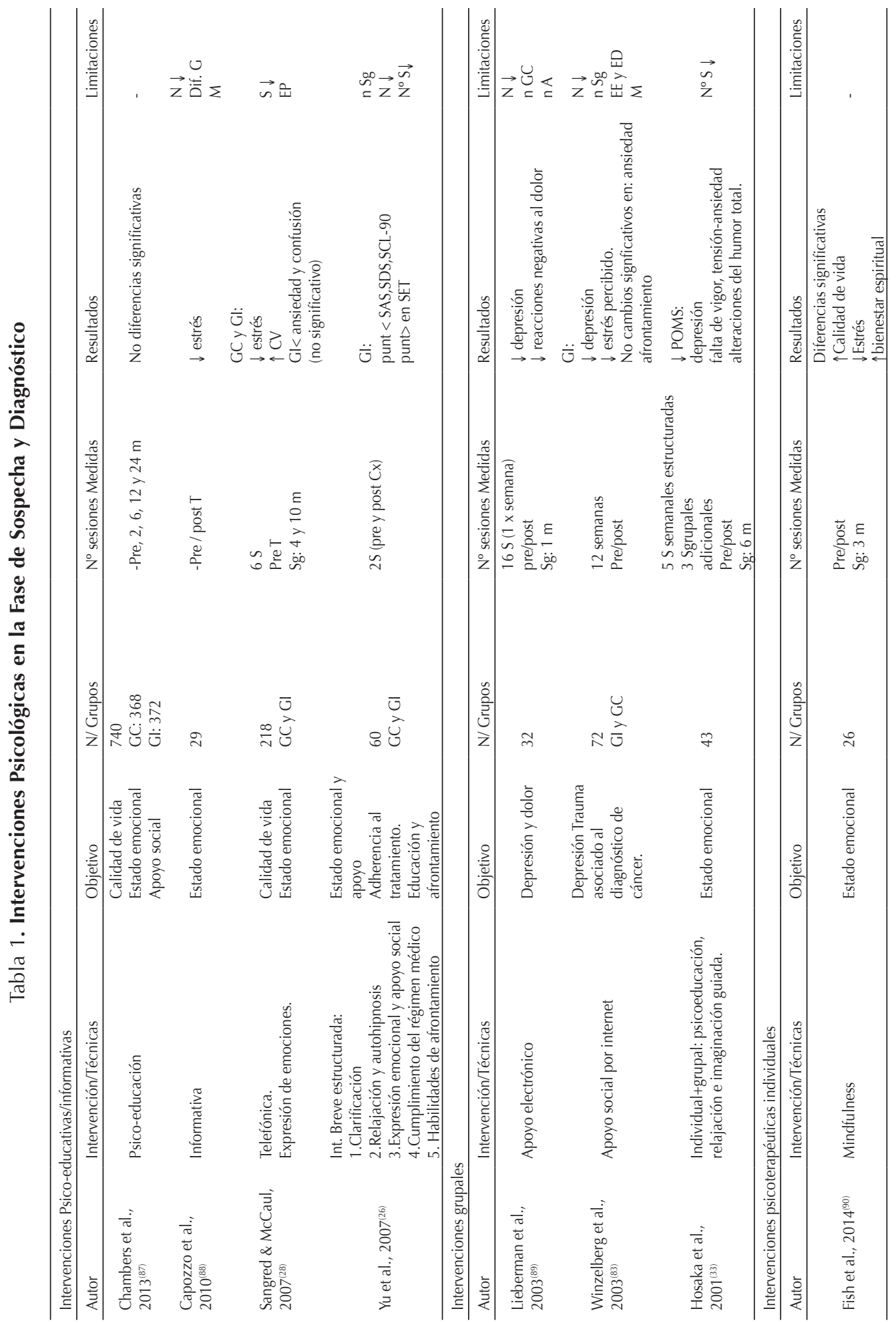




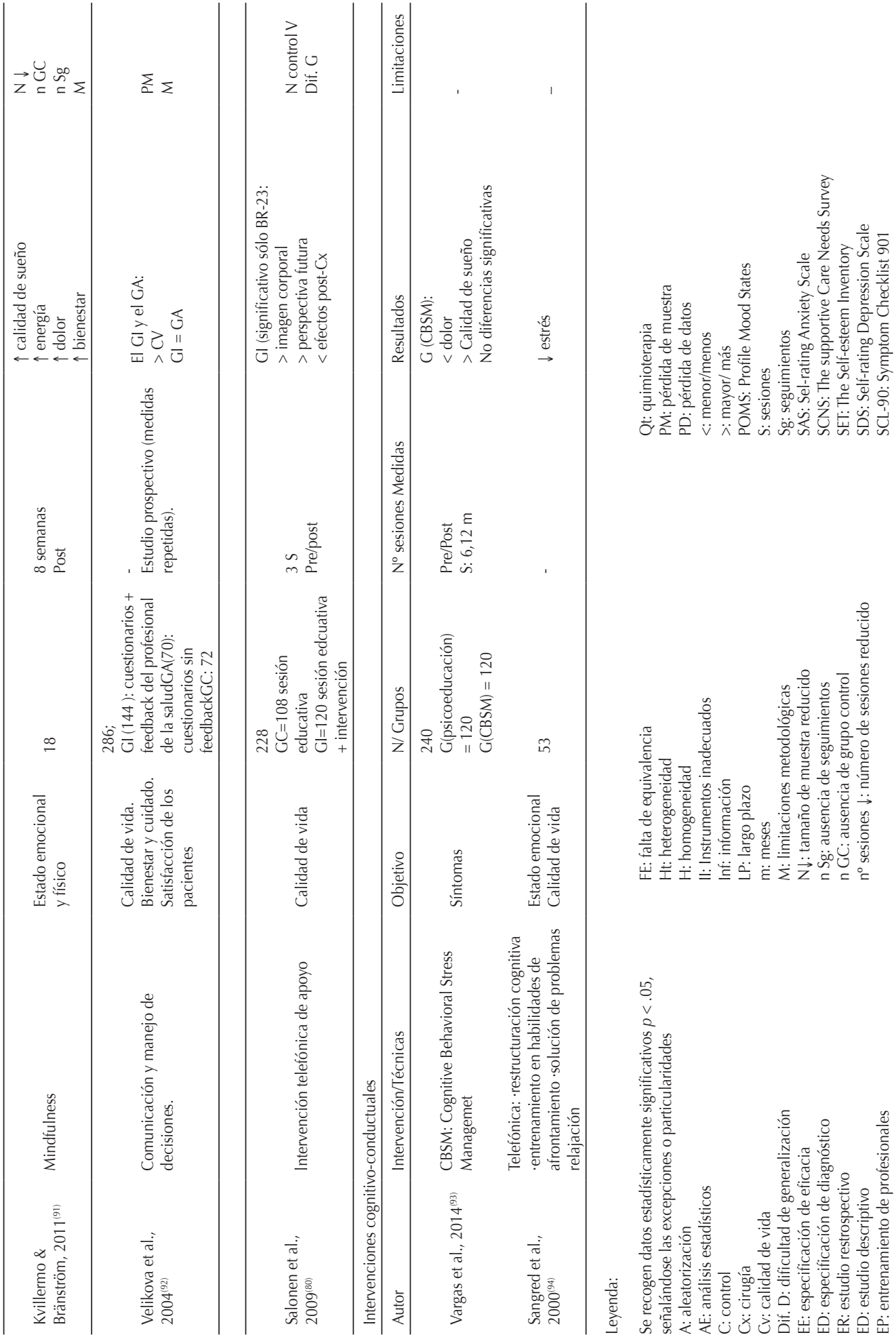


en el control de síntomas y, en general, en la mejora de la calidad de vida (véase tabla 1). También, intervenciones mediante Mindfulness señalan efectos positivos en la depresión, la ansiedad, el distrés, el dolor, el sueño y la calidad de vida (véase tabla 1).

Las intervenciones grupales, mediante distintas técnicas como apoyo social, expresión, psico-educación y Counseling, han logrado mejorar el estado afectivo (reducción de ansiedad, estrés, depresión), el ajuste y el conocimiento sobre la enfermedad (véase tabla 1).

A pesar de que todos los estudios revisados señalan resultados favorables, no siempre han resultado concluyentes, presentado con frecuencia importantes limitaciones. Por un lado, la presencia de diferentes problemas metodológicos como reducido tamaño muestral, ausencia de aleatorización, número de sesiones reducido, indefinición de variable dependiente/independiente, ausencia de grupo control, representatividad de las muestras, etc. Por otro lado, en algunos trabajos se han empleado técnicas no siempre justificadas por los objetivos de la intervención.

Revisión de intervenciones psicológicas en la fase de tratamiento

Con respecto a las intervenciones psico-educativas se observan resultados positivos en distintos aspectos. Diferentes trabajos, mediante sesiones de educación/información lograron un mejor estado emocional, niveles más altos de satisfacción con la información y mejoras en el control del dolor. En cambio, otros trabajos mediante intervenciones similares, se muestran menos exitosos en el manejo del dolor, aunque mejoran el conocimiento sobre el mismo (véase tabla 2). En relación con este último, el trabajo de Pruitt et al. (1993), sobre afrontamiento y habilidades de comunicación en pacientes bajo radioterapia, muestra resultados contrarios, no hallándose mejoras a nivel de conocimiento ${ }^{(37)}$.

En relación con el estado afectivo, la educación para el manejo de síntomas y las estrategias de afrontamiento, el Counseling, el apoyo y la relajación parecen reducir la ansiedad, la depresión, la ira, así como mejorar el funcionamiento y la calidad de vida (véase tabla 2).

Desde la perspectiva cognitivo-conductual algunas de las técnicas conductuales utilizadas de forma aislada o combinada, se destinaron a objetivos como el control de síntomas, la mejora del estado emocional y el funcionamiento del paciente (véase tabla 2). Diferentes estudios han mostrado resultados favorables, especialmente en relación con las náuseas-vómitos, el dolor y el cansancio. Para el manejo del dolor, más concretamente, técnicas como la reestructuración cognitiva ${ }^{(38)}$ o la relajación ${ }^{(39)}$ mostraron resultados positivos al igual que la hipnosis ${ }^{(40)}$. Los estudios que mediante relajación e hipnosis ${ }^{(41,42)}$ se focalizaron en náuseas y vómitos anticipatorios en pacientes en quimioterapia o mediante la desensibilización sistemática o distracción cognitivoconductual ${ }^{(43)}$, lograron evitar su aparición. En cuanto a náuseas y vómitos post-tratamiento se observaron resultados favorables en relación con el control de su intensidad pero no de su evitación (véase tabla 2). Las estrategias conductuales llevadas a cabo para el control del cansancio han tenido como objetivos fomentar estrategias de autocuidado saludables, como la administración de tiempo y energía, el mantenimiento del apoyo y contacto con el entorno social y familiar ${ }^{(44)}$. Además, programas de ejercicio físico han obtenido resultados satisfactorios acompañados de beneficios físicos, emocionales, sociales y en la calidad de vida global (véase tabla 2). En esta línea apunta el meta-análisis de Carayol et al. (2015) sobre intervenciones basadas en ejercicio en pacientes con cáncer de mama durante la quimioterapia y la radioterapia ${ }^{(45)}$. 
Con respecto al estado emocional, los trabajos han tratado principalmente, la reducción del estrés, ansiedad y depresión y mejora de la calidad de vida (véase tabla 2).

Por último, algunas de estas intervenciones también trataron de facilitar la rehabilitación y el funcionamiento global del paciente, con la consiguiente recuperación o mantenimiento del mayor número de actividades posibles, sin olvidar sus limitaciones y el nivel de actividad previo (véase tabla 2). Trabajos anteriores en esta dirección señalaron una menor estancia hospitalaria tras la cirugía mediante hipnosis e imaginación guiada ${ }^{(46)}$ y mejoras en el funcionamiento social y sexual, así como en habilidades de afrontamiento mediante relajación muscular progresiva, control y expresión de las emociones y técnicas de afrontamiento ${ }^{(30)}$.

Las intervenciones psicoterapéuticas individuales revisadas, mediante terapia de autoayuda logran la reducción y/o control de síntomas ${ }^{(47)}$. Mediante intervención telefónica muestran efectos positivos en el estado de ánimo negativo (ansiedad, depresión) y estrés ${ }^{(30,48)}$. El trabajo de Maguire et al. (1983), mediante Counseling parece mejorar el nivel de funcionamiento general y la adaptación, así como la calidad de vida(49), aun cuando los hallazgos de algunos estudios apunten a resultados no tan satisfactorios, no habiendo mejora en la calidad de vida, ni en el funcionamiento psicosocial ${ }^{(50)}$. Por otro lado, Jahn et al. (2014), utilizando Counseling, además de entrenamiento en habilidades y educación, obtienen diferencias significativas con respecto al manejo del dolor ${ }^{(51)}$.

Por último, las intervenciones basadas en Mindfulness también parecen señalar mejoras significativas en el estado emocional (ansiedad y depresión) de los pacientes (véase tabla 2).

Las intervenciones grupales indican resultados positivos en la mejora del estado de ánimo, control de síntomas y mejora del conocimiento sobre la enfermedad y el tratamiento y de la calidad de vida global. En la mayoría de ellos se trabaja el apoyo social y emocional, afrontamiento y relajación (véase tabla 2).

Los trabajos expuestos en esta fase, obtienen resultados que, en general, parecen apoyar su aplicación. No obstante, se observa como en algunos de ellos hubo problemas por falta de especificidad entre los paquetes de técnicas de intervención utilizados y los objetivos del estudio. Además, estos trabajos, al igual que los planteados en la fase anterior, no dejan de presentar distintas limitaciones metodológicas que comprometen la validez de las conclusiones obtenidas.

Revisión de intervenciones psicológicas en la fase libre de enfermedad/supervivencia

Las intervenciones encaminadas a proveer de apoyo e información parecen favorecer la recuperación y manejo de la actividad laboral durante o después de la enfermedad y/o tratamiento, destacando además el papel que puede desempeñar el equipo médico y el lugar de trabajo (véase tabla 3). La revisión de Hoving et al. (2009) puso de manifiesto la necesidad de seguir desarrollando trabajos encaminados a este tipo de intervención, con el fin de consolidar estas afirmaciones ${ }^{(15)}$. También otros trabajos, usando este tipo de intervenciones señalan mejoras en el estado emocional, síntomas físicos y calidad de vida de los supervivientes (véase tabla 3).

Mediante técnicas conductuales y ejercicio físico, el meta-análisis de Dujits et al. (2011), o los trabajos de Penttinen et al. (2011) y Schmitz (2011) indicaron los posibles beneficios para los supervivientes en relación con alteraciones emocionales (ansiedad, depresión y estrés), síntomas (cansancio), imagen corporal y calidad de vida(52-54). De igual modo, Rogers et al. (2011) resaltaron la importancia de dis- 


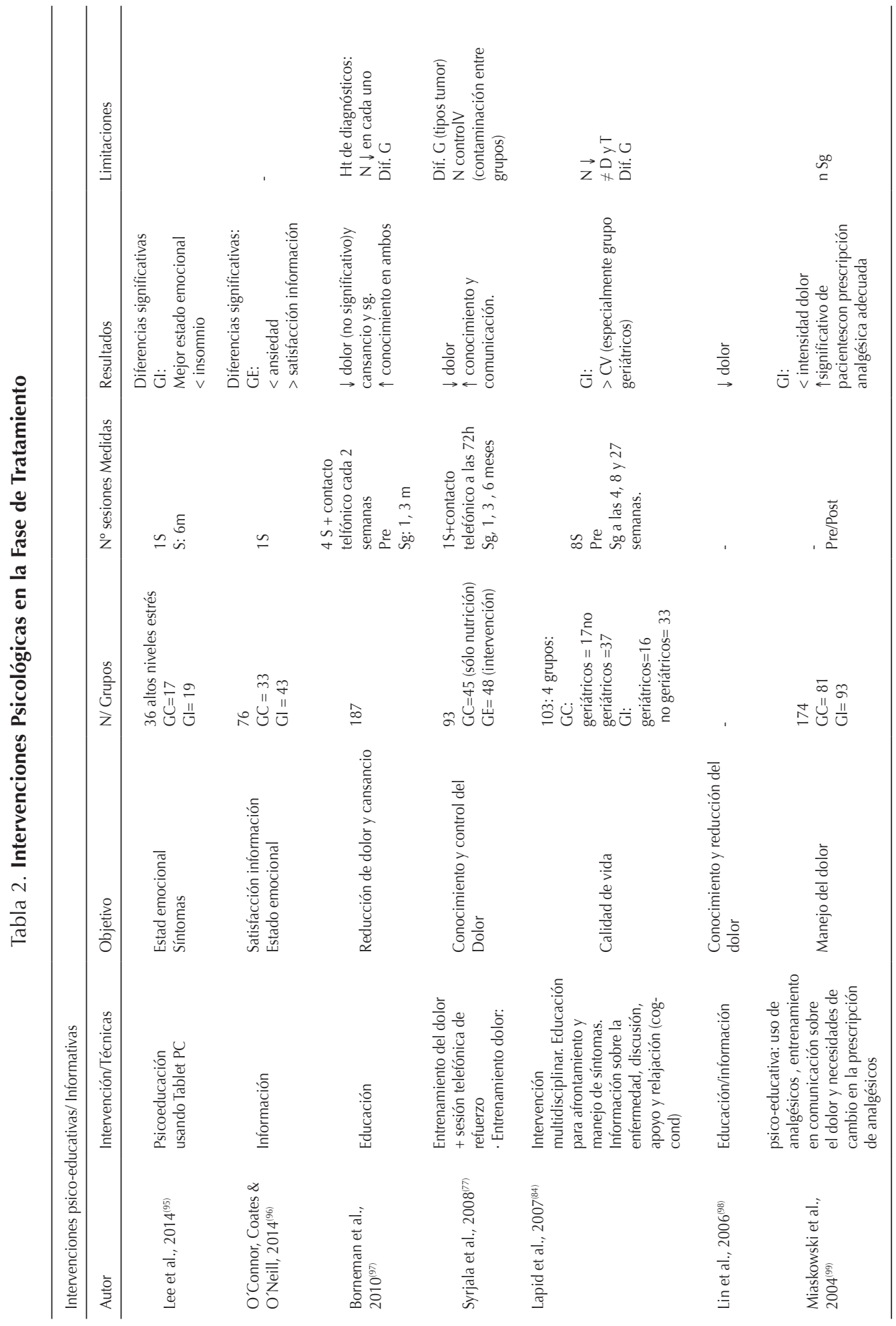




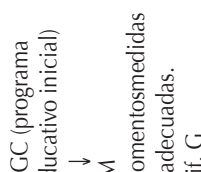

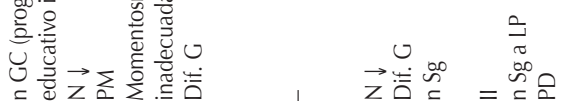

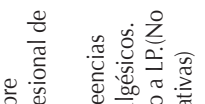

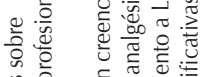

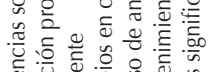

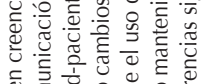

ᄂ.

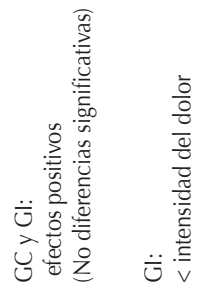

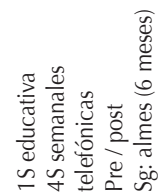

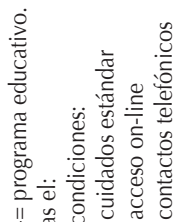

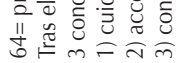

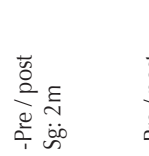

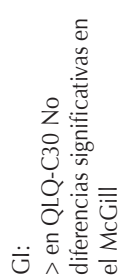

تิ

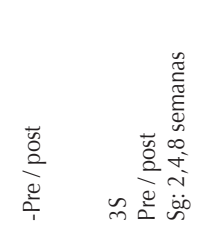
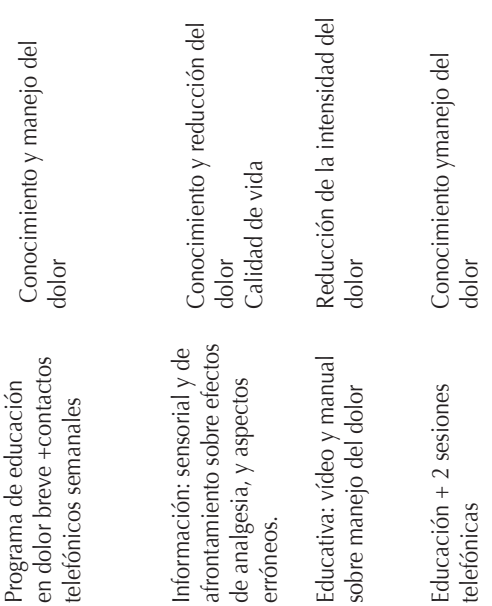

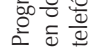

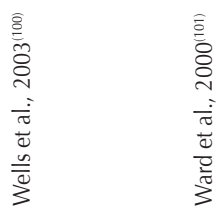

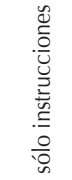

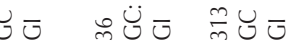

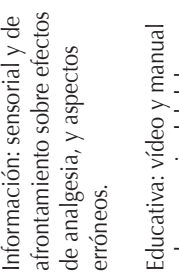

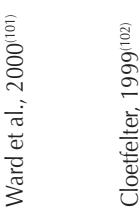

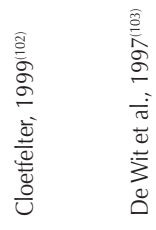

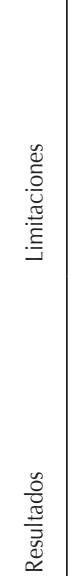

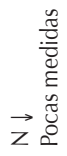

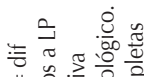

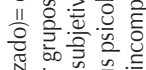

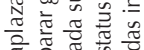

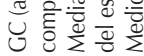

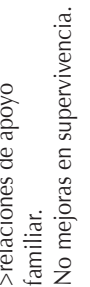

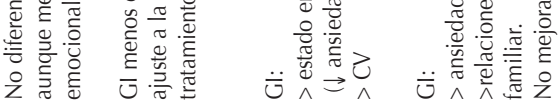

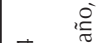

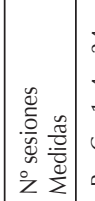

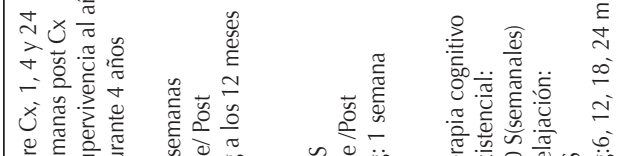

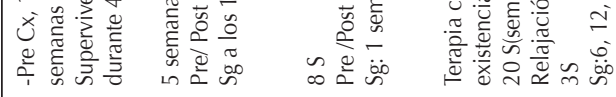

은

৪ نिं

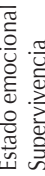

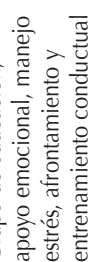

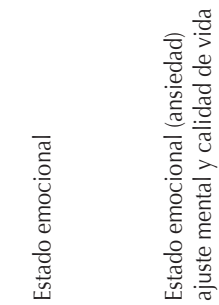

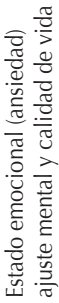
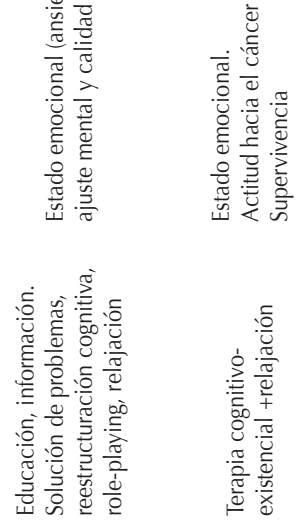

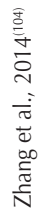

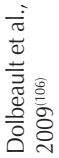

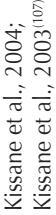




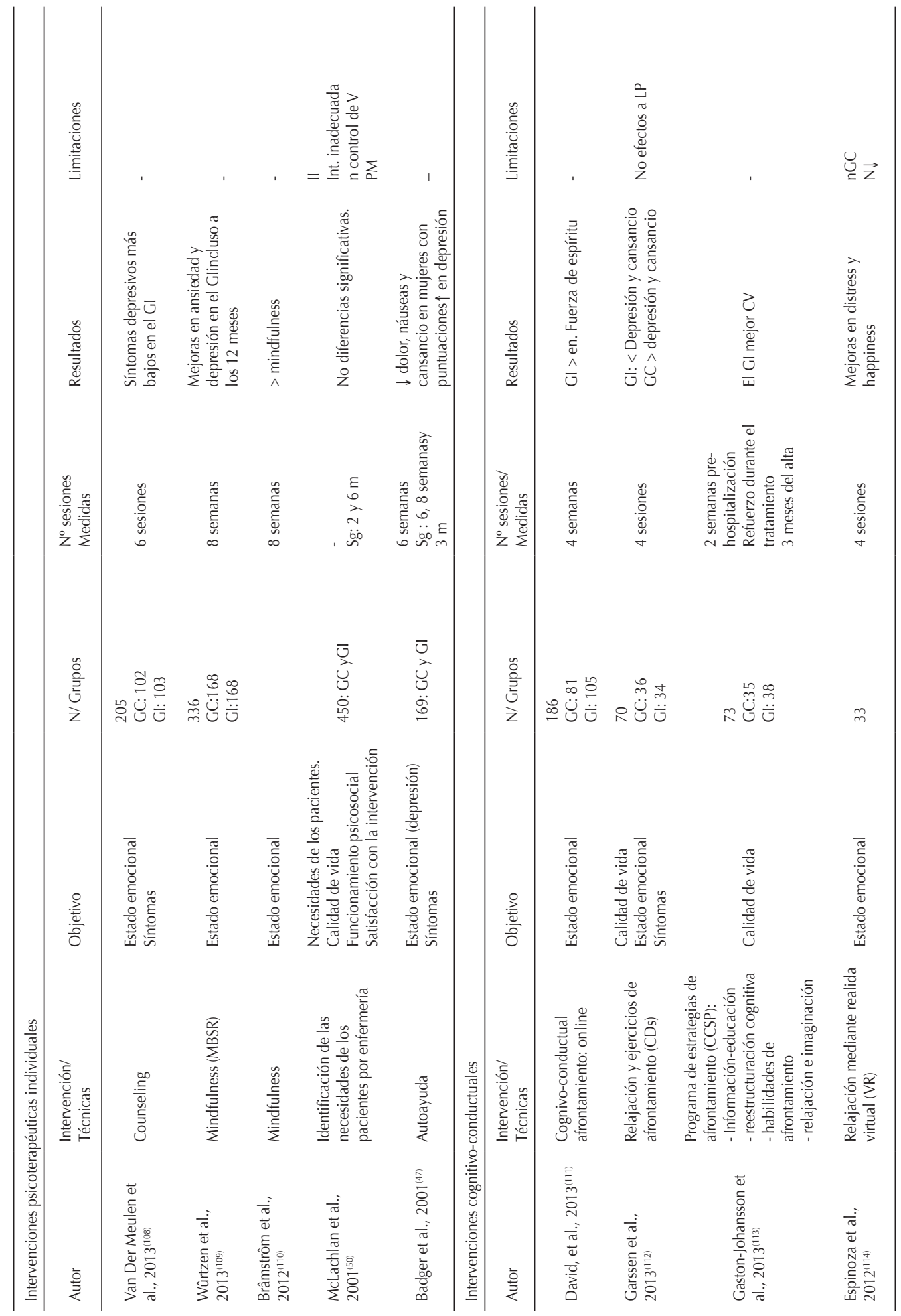




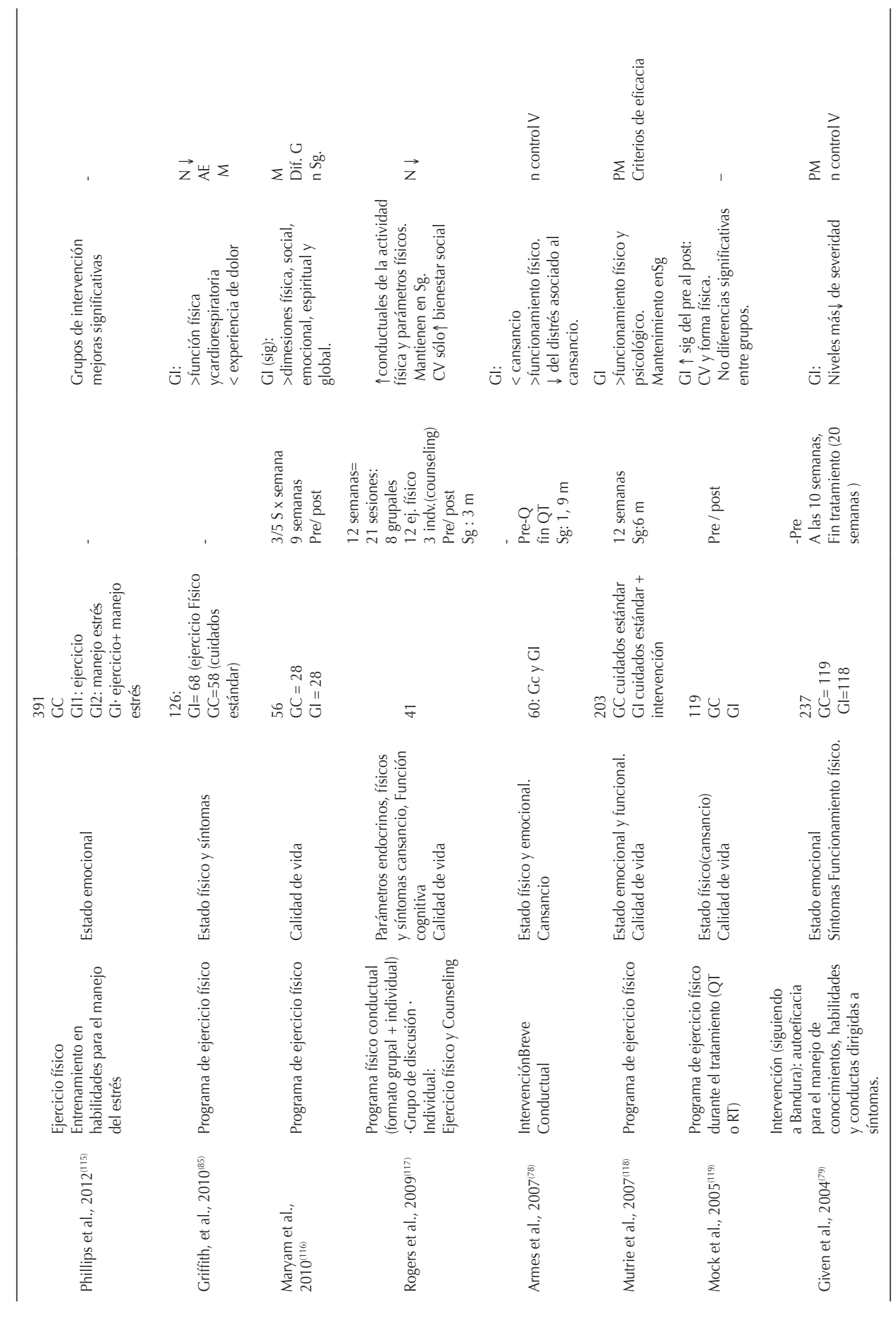


도은

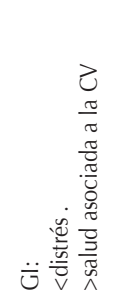

$\stackrel{\infty}{z}$

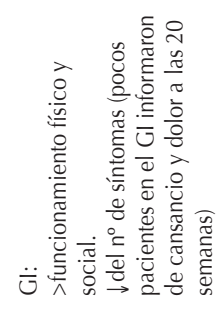

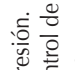

造起

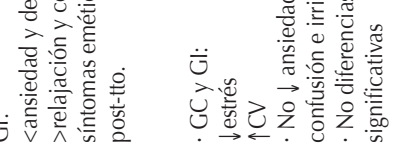

$\sqrt{\frac{y^{5}}{2}}$

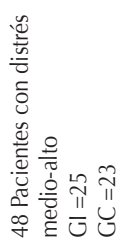

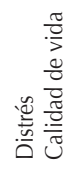

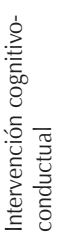

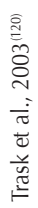

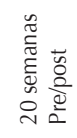
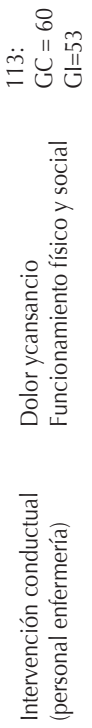

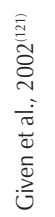

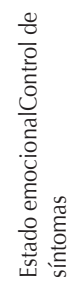

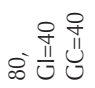

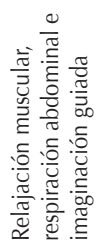

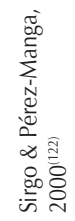

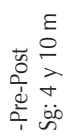

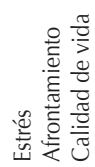

$\Re \cup \cup$
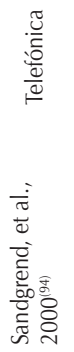
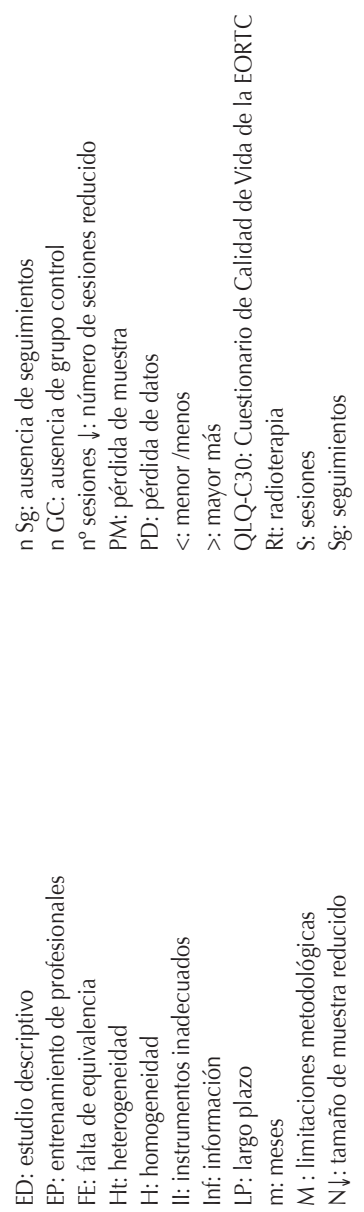

เุำ

v

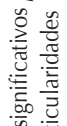

吾

悹

悾

竞

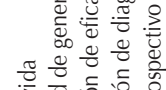

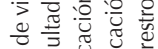

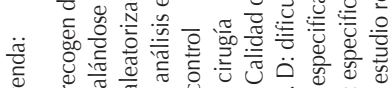

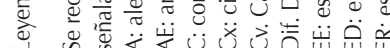


tintos patrones de actividad física para la mejora de síntomas físicos, como el cansancio, y de actividades de tiempo libre para síntomas depresivos ${ }^{(55)}$.

Con respecto a las intervenciones psicoterapéuticas individuales relacionadas con el Mindfulness, se observó una mejora en la calidad de vida y reducción del estrés y discapacidad. En la misma línea, trabajos basados en la reducción del estrés obtuvieron mejoras en el estado emocional, síntomas físicos y en algunos parámetros fisiológicos (véase tabla 3).

Desde las terapias grupales, Ochoa et al. (2010) pusieron en marcha una intervención basada en psicoterapia positiva con el objetivo de favorecer la adaptación psico-social del paciente oncológico en esta fase. Se trataba de reducir emociones negativas, trabajando emociones positivas, la regulación emocional y el afrontamiento y facilitar el crecimiento postraumático ${ }^{(56)}$. Tras la intervención se logró una reducción significativa de la sintomatología postraumática, manteniéndose e incluso incrementándose los efectos de reducción emocional en el seguimiento a los 3 meses. Thompson et al. (2014) mediante una intervención para facilitar el proceso de transición de la enfermedad a la fase libre de la misma obtuvieron resultados favorables en relación con sentir más apoyo e incremento de la confianza; sin embargo, no hallaron diferencias significativas en cuanto al estado emocional ${ }^{(57)}$.

Con respecto a síntomas físicos, Fillion et al. (2008) lograron resultados positivos en relación con cansancio, nivel de energía, distrés emocional y calidad del funcionamiento físico mediante una intervención psicológica breve para el manejo del cansancio $^{(58)}$. Otros estudios señalaron cómo intervenciones de carácter educativo-informativo podrían contribuir con la mejora de limitaciones físicas derivadas del tratamiento, específicamente, al linfedema con la consiguiente mejora de la calidad de vida ${ }^{(59)}$.
A pesar de la identificación de los distintos problemas que los pacientes "libres de enfermedad" pueden experimentar y de la posible adecuación de la intervención psicológica, la revisión señala cómo, en general, son escasos los estudios que se han centrado en la intervención orientada exclusivamente en esta fase. Esto, puede suponer un obstáculo a la hora de establecer el papel que la intervención psicológica puede desempeñar con estos pacientes. No obstante, parece que las investigaciones realizadas presentan resultados favorables, sin olvidar la presencia de las limitaciones metodológicas (ausencia de seguimientos, tamaño de muestra reducido, problemas de representatividad de las muestras, ausencia de grupo control, etc.) además de la necesidad de especificar las técnicas más adecuadas para cada condición.

Efectos de la intervención psicológica en la supervivencia

Otro aspecto investigado en cáncer, es el posible papel que la intervención psicológica puede desempeñar sobre el sistema inmune, así como en la supervivencia de los pacientes oncológicos e incluso en el riesgo a una recaída ${ }^{(5,7,60,61)}$. Con distintos tipos de intervención, tales como grupos psico-educativos y de apoyo, la meditación e imaginación, el uso de técnicas de reducción de estrés, promoción de conductas de salud y mantenimiento y adherencia al tratamiento se observaron incrementos en la supervivencia ${ }^{(62-73)}$. Los datos evidencian de los posibles beneficios de la intervención psicológica en distintos parámetros biológicos, en el incremento de la supervivencia y en la reducción en el riesgo de recaída. Sin embargo, estos trabajos no dejan de presentar importantes limitaciones que ponen en duda la claridad de los resultados obtenidos. Esto queda reflejado también en otros trabajos de revisión ${ }^{(5,64,74)}$. 


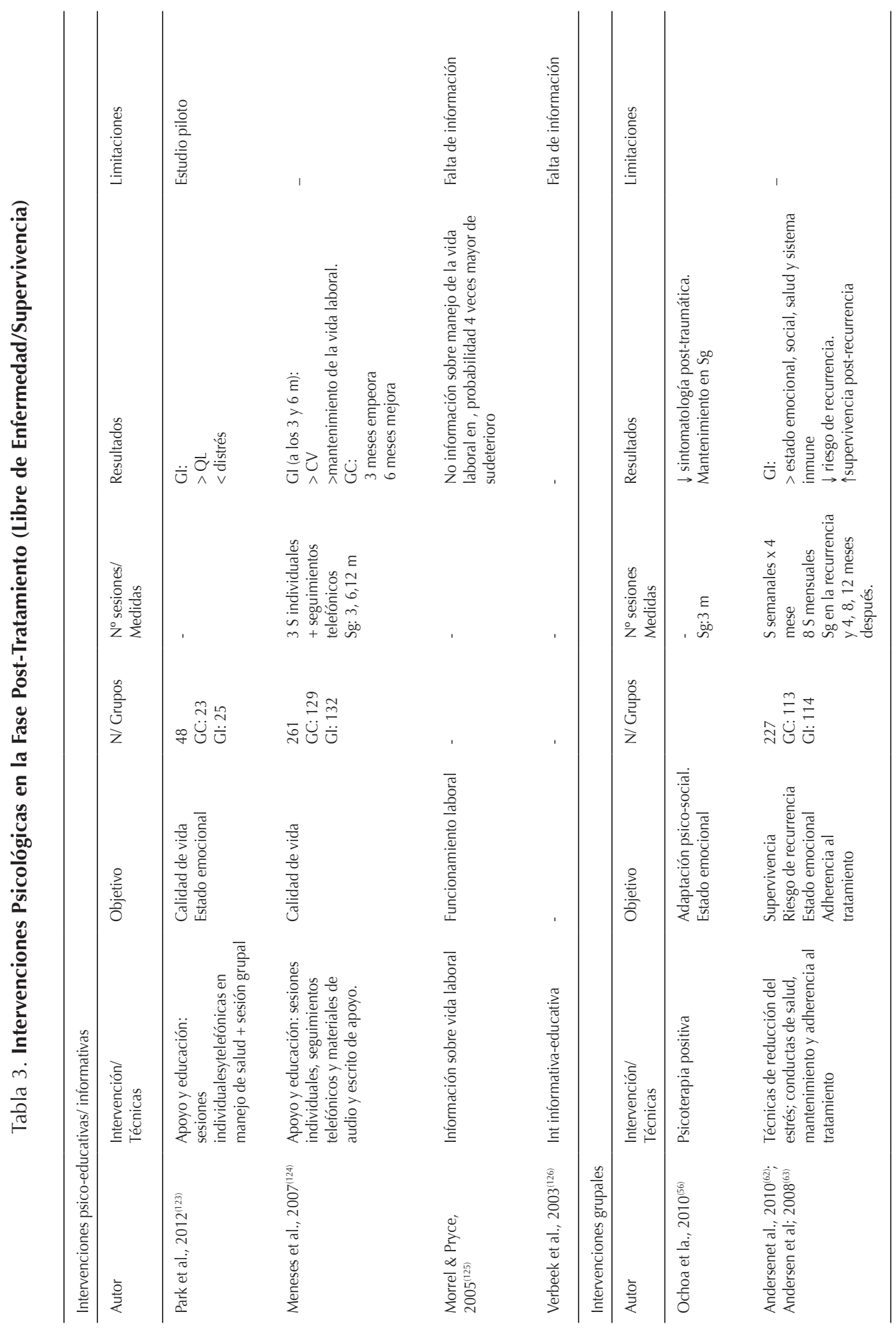




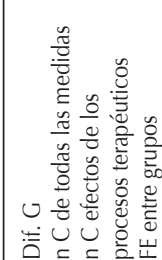

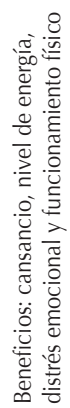

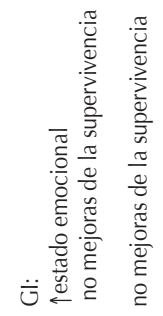

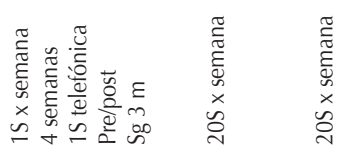

学

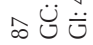

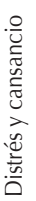

品守学 ลิ心

$\circ$

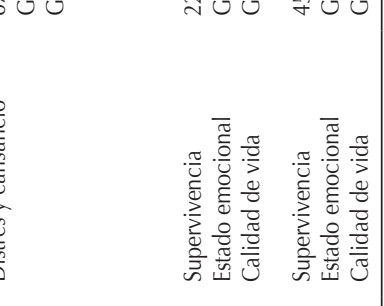

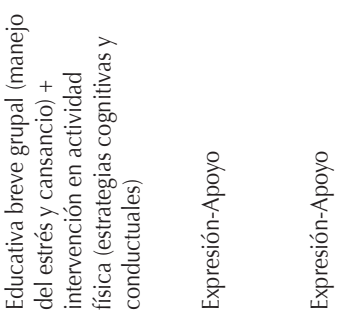

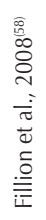

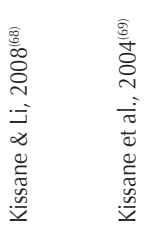

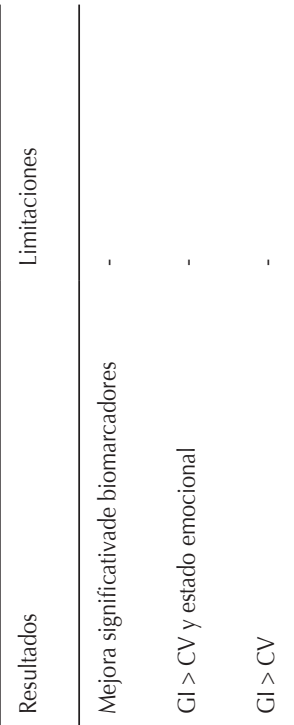

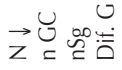
$\vec{z} \Sigma$<smiles>[C+]1C=C[c+]2cc[c+]21</smiles>
क्ष

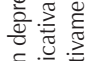

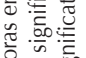

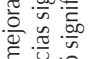

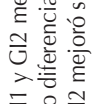

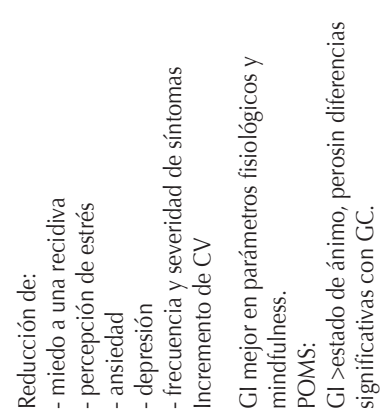
ज艺<smiles>C1C[C@H]2C[C@H]1[C@@H]1CC[C@H]21</smiles>
, 올 z ₹ ฟิ $\infty \cup$

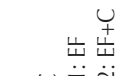

古 드느 긴

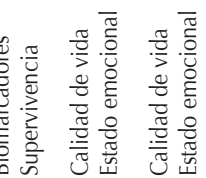

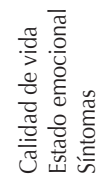

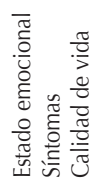

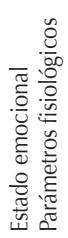

$\frac{\stackrel{2}{\frac{0}{2}}}{0}$

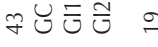

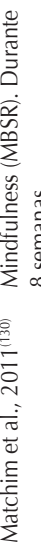




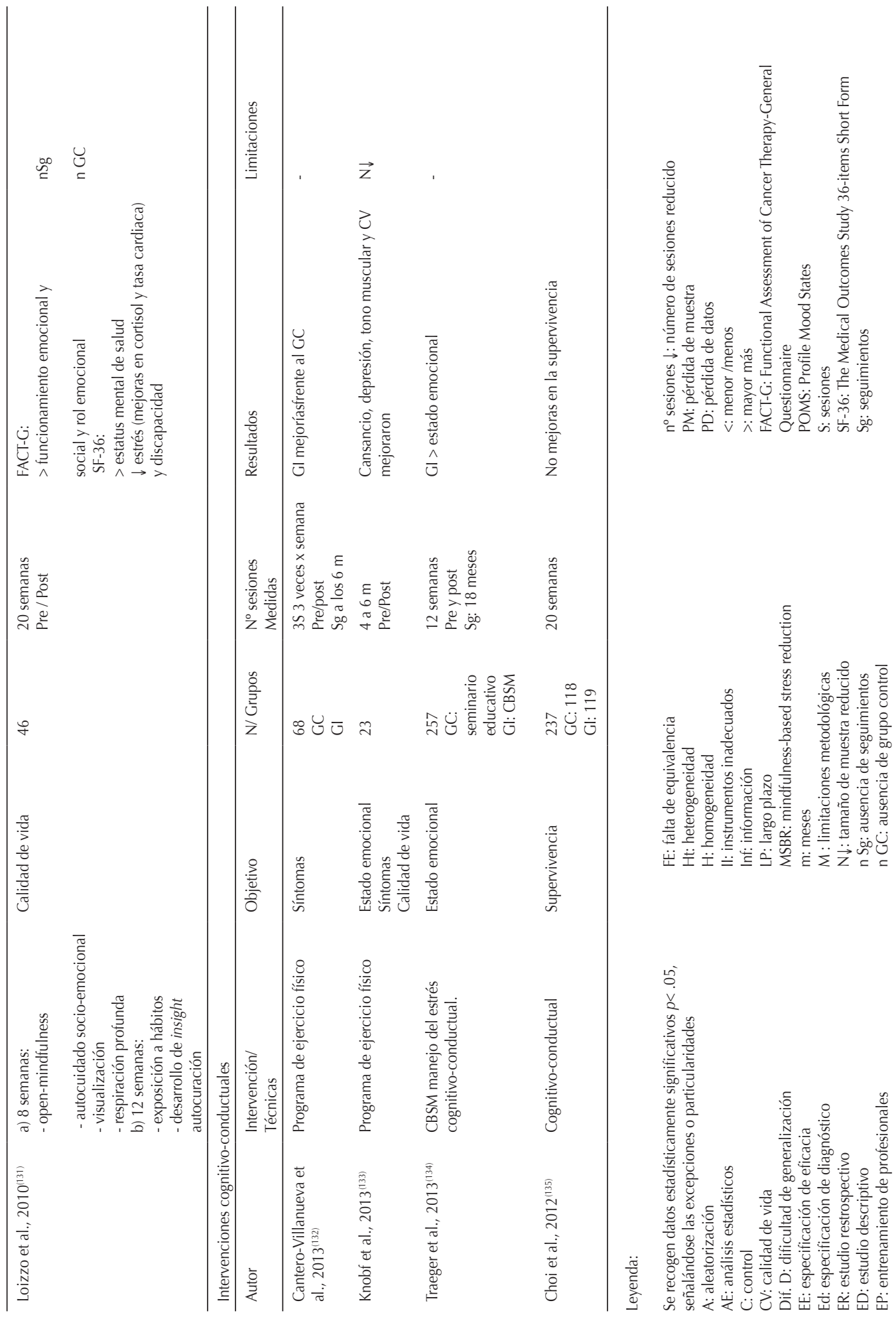


Revisión de intervenciones psicológicas en la fase de recidiva, progresión y enfermedad terminal

Desde la literatura se ofrece como distintos tipos de intervención psicológica pueden favorecer el ajuste y el afrontamiento a la enfermedad, la reducción del estrés, la mejora en la autoestima y el estado emocional, el control de síntomas y, en definitiva, la calidad de vida del paciente.

En las intervenciones cognitivo-conductuales para la mejora del estado emocional se observan reducciones significativas de ansiedad y depresión. En las intervenciones mediante grupos de apoyo, educativos y expresión emocional, la mejora del estado afectivo, afrontamiento de la enfermedad, y control de síntomas.

También se han Ilevado a cabo intervenciones psico-educativas e intervenciones psicoterapéuticas individuales con objetivos y resultados similares (véase tabla 4).

Las intervenciones psicoterapéuticas individuales se han destinado a fomentar una relación de empatía y comprensión ${ }^{(32)}$, con el objetivo de facilitar el apoyo emocional. En general, con los pacientes oncológicos esta terapia se centró más en esta relación que en la aplicación de técnicas específicas. Se trata por lo tanto de mejorar su estado afectivo y tratar de disminuir el estrés y la ansiedad que el paciente pueda estar experimentando ante esta situación.

Cuando la enfermedad comienza su progresión, mejorar el estado afectivo, controlar los síntomas como consecuencia de la progresión de la enfermedad, así como mejorar su manejo y facilitar el soporte emocional familiar, la comunicación y la resolución de conflictos, suponen los objetivos centrales de la intervención. Se trata en definitiva, de preservar y/o mejorar la calidad de vida del paciente (véase Tabla 4).

Desde el enfoque cognitivo-conductual, los estudios muestran resultados fa- vorables en la reducción de la depresión, la ansiedad, el cansancio y el distrés general, el afrontamiento a la enfermedad, el autocuidado y la calidad de vida (véase tabla 4).

Mediante grupos de apoyo se logra la reducción de alteraciones afectivas y síntomas y un mejor afrontamiento a la enfermedad (veáse tabla 4).

Por otro lado, algunas intervenciones psicoterapéuticas individuales muestran su eficacia para reducir el estrés y mejorar la calidad de vida ${ }^{(70,75,76)}$.

En general, parece que las estrategias puestas en marcha en esta fase señalan efectos positivos en algunos aspectos relacionados con el estado emocional y físico. Así, Fawcy (1999) señala que estas estrategias pueden, en ocasiones, ayudar a trabajar más fácilmente el auto-duelo y el dolor emocional logrando en lo posible el mejor control de los síntomas y preservando un nivel de calidad de vida que permita que la muerte se sobrelleve mejor ${ }^{(9)}$.

Los trabajos presentados en esta fase también conllevan importantes limitaciones: de nuevo, la falta de adecuación entre los objetivos y las técnicas dirigidos a ellos junto con los problemas metodológicos.

\section{DISCUSIÓN/CONCLUSIONES}

En general, en todas las fases se ha subrayado la importancia de distintos aspectos como fomentar la relación médicopaciente, proporcionar información clara y adecuada, ofrecer apoyo y tratar de mejorar el estado emocional, físico y funcional de los pacientes. Así, se trataría de reducir la incertidumbre, facilitar la toma de decisiones y mejorar el ajuste a la enfermedad y, sobre todo, favorecer la calidad de vida del paciente.

Los trabajos revisados, aunque a veces con resultados contradictorios, se han centrado en objetivos relacionados con la mejora del estado emocional, el control 


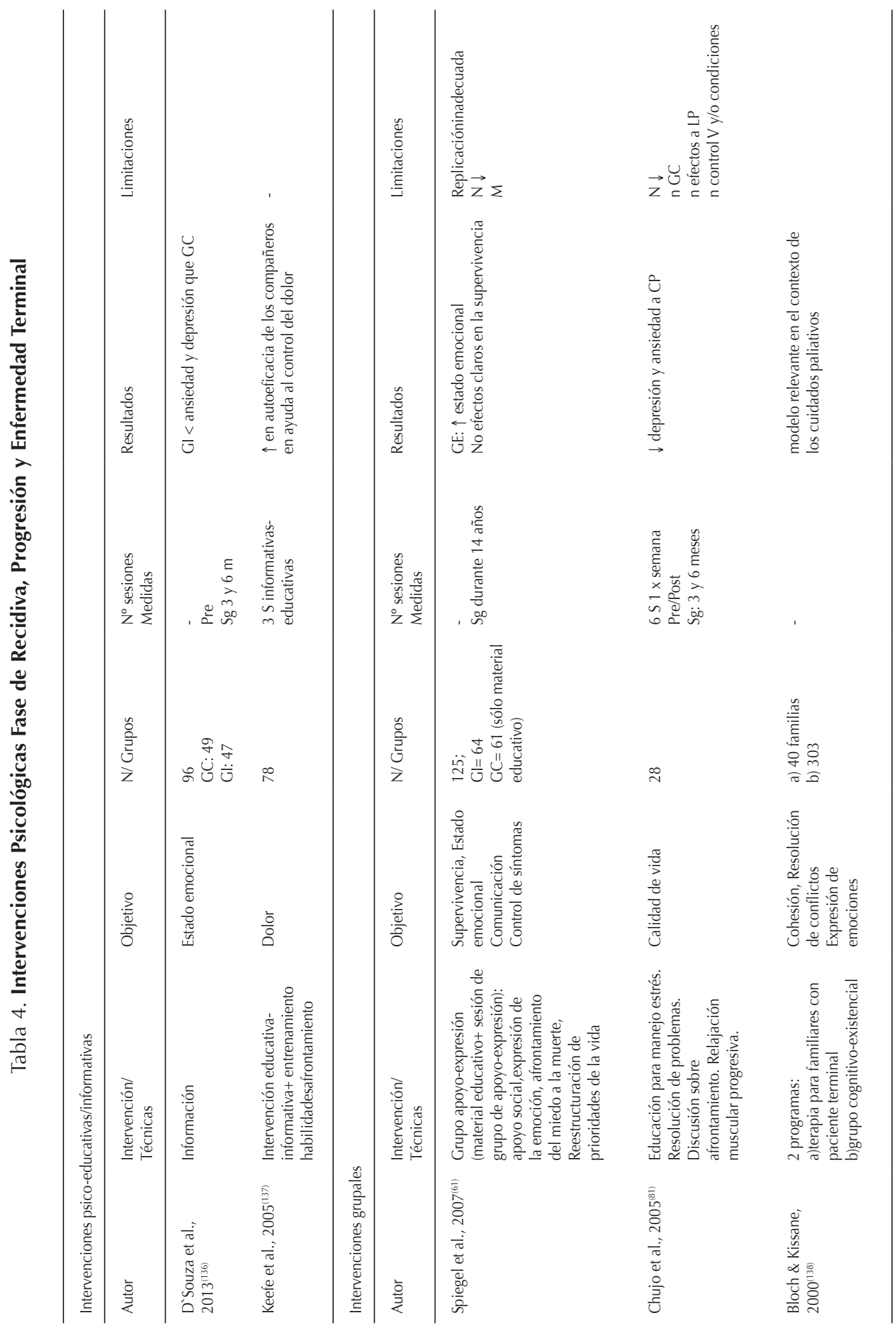




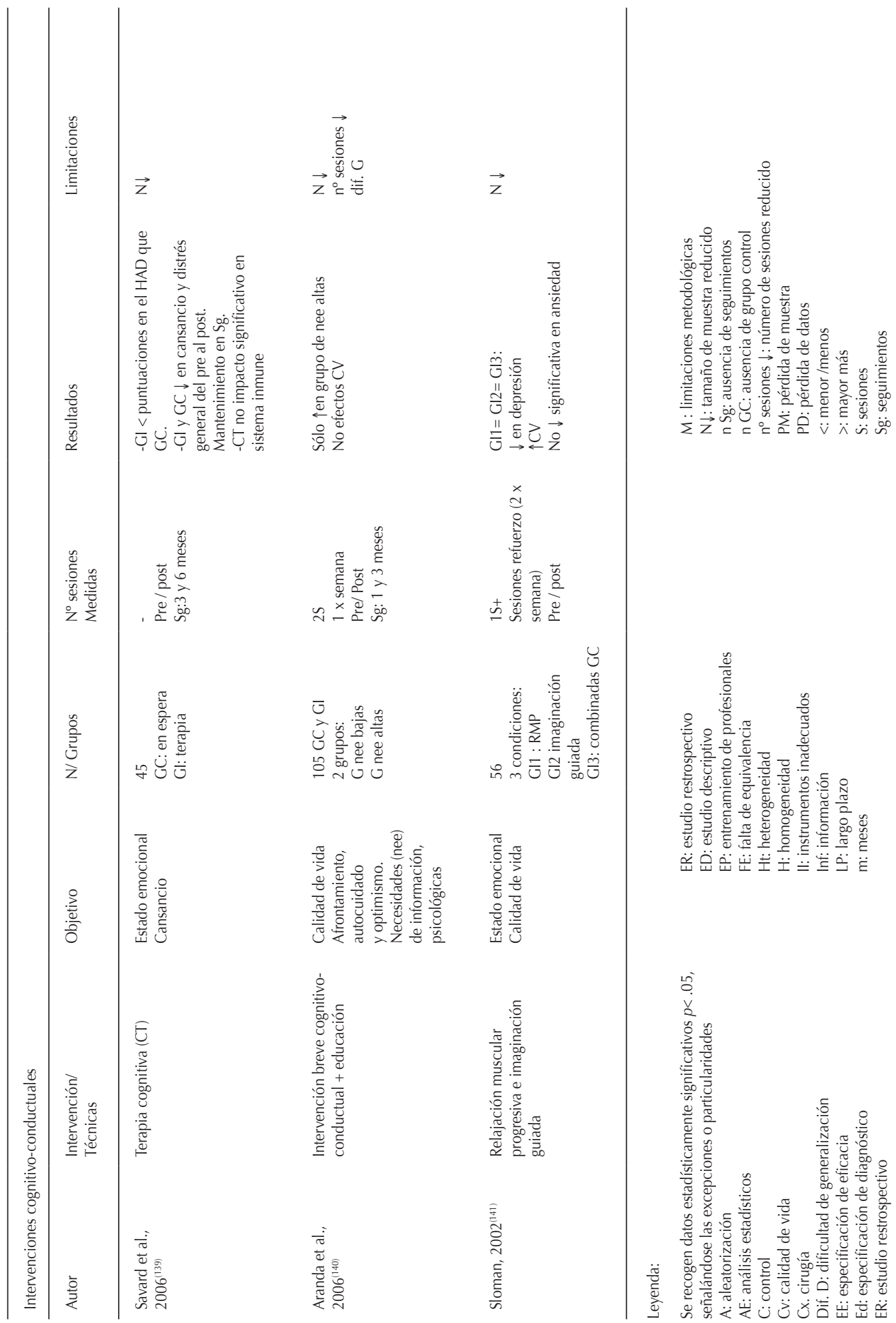


de síntomas asociados con el tratamiento y proceso de enfermedad, mejora y recuperación del estatus funcional de los pacientes y en la calidad de vida. En general, las diferentes intervenciones han logrado resultados favorables acorde con sus objetivos lo que recomendarían su aplicación. Distintas revisiones realizadas en los últimos años apuntan en esta dirección, a pesar de la utilización de diferentes criterios de evaluación y clasificación. La revisión de Newell et al. (2002), centrándose en trabajos con garantías metodológicas los clasifica en función de los objetivos de la intervención ${ }^{(7)}$. Aunque enfatiza en la necesidad de tener cautela, a su vez reconoce sus potenciales beneficios. Especialmente destacan la terapia grupal, la intervención educativa, el Counseling y la terapia cognitivo-conductual, así como sus posibles efectos a mediano y largo plazo. El trabajo de Barsevick et al. (2002) revisa intervenciones, aunque sólo dirigidas a la depresión, obteniendo resultados favorables mediante intervenciones similares ${ }^{(10)}$. Blake-Mortimer et al. (1999) exploran la efectividad de la psicoterapia grupal en la calidad y cantidad de vida. Señalan como distintas terapias de grupo reducen trastornos afectivos, el dolor, mejoran la calidad de vida del paciente oncológico y, aunque sin resultados concluyentes, exponen trabajos que pueden tener efectos positivos en la cantidad de vida ${ }^{(5)}$.

No obstante, algunos trabajos aquí expuestos han empleado técnicas no siempre justificadas por los objetivos de la intervención, bien, no cuentan con estudios u hallazgos previos de las mismas características que las avalen o porque aun existiendo estos, no han cumplido los requisitos necesarios para proporcionar las garantías metodológicas que apoyen su utilización. En general, muchos trabajos mediante la aplicación de paquetes de intervención estandarizados, se han centrado en numerosas y complejas conductas con funcio- nalidades diferentes, atendiendo más a las topografías que a la función que desempeñan, sin considerar que las condiciones que las controlan pueden ser distintas.

La ausencia de especificidad entre técnicas y objetivos, supone una dificultad para establecer las condiciones en las que una intervención puede resultar de utilidad y para qué pacientes puede ser más adecuada.

La revisión de Lepore y Coyne (2006) ${ }^{(8)}$ que recoge las revisiones anteriormente mencionadas, pone de manifiesto las diferencias entre las mismas a la hora de concluir sobre la efectividad y los beneficios de la intervención psicológica en cáncer ${ }^{(8)}$. Señalan que las revisiones más rigurosas presentan limitaciones en la selección de trabajos que reúnan garantías metodológicas y aquellos que obtienen resultados que apoyan la eficacia de la intervención psicológica. Asimismo, ponen en duda las evidencias arrojadas sobre la adecuación de la intervención psicológica en la reducción del estrés en pacientes con cáncer, cuestionando los beneficios de su aplicación.

Por todo ello, se requiere precaución en la consideración de las conclusiones de los trabajos realizados. Es necesario tener en cuenta que estas investigaciones, independientemente de la fase de la enfermedad en la que se han llevado a cabo, de su modalidad y objetivos, no han dejado de presentar distintas limitaciones metodológicas, que también han quedado patentes en los trabajos de revisión ya comentados. Ibáñez y Soriano (2008) ponen de manifiesto la violación de las normas estándar de la investigación científica por diferentes estudios basados en la importancia de la intervención psicológica en enfermos oncológicos ${ }^{(16)}$. Esto hace referencia a los trabajos expuestos a lo largo del presente trabajo, las limitaciones encontradas han ido en la misma línea. Se destacan, el uso de tamaños de muestra 
pequeños o la pérdida de sujetos a lo largo del tiempo, debido en muchas ocasiones, a las propias peculiaridades de la muestra, en relación con el estado físico, el pronóstico, las dificultades de acceso a la misma y a los abandonos esperables en cualquier investigación (véanse tablas 1, 2 y 4). Esto ha supuesto la pérdida de información así como dificultades a la hora de generalizar los resultados a la población. Además, se han encontrado trabajos que no cuentan con grupo control ${ }^{(11,23)}$, ausencia de un procedimiento de aleatorización adecuado $^{(77)}$, ausencia de control de las variables ${ }^{(50,61,78-80)}$; falta de equivalencia entre el grupo control y el grupo experimental en características clínicas, sociodemográficas, entre otras, dificultad para la clarificación de las condiciones que hacen más o menos efectiva una intervención ${ }^{(50,81-83)}$, uso de muestras demasiado homogéneas ${ }^{(77,84)}$ con la consiguiente dificultad de generalización, ausencia de seguimientos a largo plazo ${ }^{(83)}$, problemas con los instrumentos de medida utilizados y los análisis estadísticos ${ }^{(82,85)}$ y el uso de estudios retrospecti$\operatorname{vos}^{(86)}$. Todo ello, en muchos casos, obliga a tomar con cautela los resultados obtenidos, dificulta la comparación objetiva de los datos proporcionados por los distintos estudios, la obtención de conclusiones válidas y, por lo tanto, la generalización de los resultados con las garantías metodológicas necesarias.

Además, si a ello se unen posibles problemas de falta de especificidad de objetivos y procedimientos ya comentados, se destaca la necesidad de cuestionarse las implicaciones clínicas de los mismos. No se cuestiona el papel positivo de la intervención psicológica en los pacientes oncológicos, de hecho, se ha mostrado significativo en múltiples estudios. Sin embargo, para superar las limitaciones expuestas, se hace necesario valorar hasta qué punto el diseño de la intervención propuesto permite hacer funcionales los objetivos del trabajo para los pacientes a los que va dirigido, qué medidas y cómo han de realizarse, qué instrumentos son más adecuados, qué seguimientos deben tenerse en cuenta, etc. Con todo ello, la última cuestión que quedaría plantearse es si la intervención propuesta es necesaria para todos los pacientes o para qué pacientes podría mostrarse más adecuada. Dados los antecedentes presentados, este trabajo subraya la necesidad de diseñar intervenciones psicológicas ajustadas a las necesidades de los pacientes en función de las características de cada fase de la enfermedad. De este modo sería posible valorar cuáles de los tratamientos y, especialmente, los componentes de los mismos, así como el formato de su aplicación resultan más adecuados a las peculiaridades de cada fase de la enfermedad y, más relevante aún, a las necesidades y características de los pacientes. Esto facilitaría el uso de criterios comunes en la valoración de la eficacia de las intervenciones psicológicas permitiendo obtener resultados más concluyentes.

\section{REFERENCIAS BIBLIOGRÁFICAS}

1. Fallowfield LJ. Treatment decision-making in breast cancer: The patient-doctor relationship. Breast Cancer Res Treat 2008;112(Suppl. 1):5-13. Doi: 10.1007/ s10549-008-0077-3.

2. Montazeri A. Health-related quality of life in breast cancer patients: A bibliographic review of the literature from 1974 to 2007. J Exp Clin Canc Res 2008;27(1):32. Doi: 10.1186/1756-9966-27-32.

3. Shepherd KL, Fisher SE. Prospective evaluation of quality of life in patients with oral and oropharyngeal cancer: From diagnosis to three months post-treatment. Oral Oncol 2004;40:751-7. Doi: 10.1016/j.oraloncology.2004.01.018.

4. Yokoyama T, Kurokawa Y, Kani R, Takatori E, Nokiguchi S, Suzuki Y, et al. Assessment 
of health-related quality of life in cancer outpatients treated with chemotherapy. Jpn J Cancer Chem 2012;39:409-14.

5. Blake-Mortimer J, Gore-Felton C, Kimerling R, Turner-Cobb JM, Spiegel D. Improving the quality and quantity of life among patients with cancer: A review of the effectiveness of group psychotherapy. Eur J Cancer 1999;35:1581-6. Doi: 10.1016/ S0959-8049(99)00194-X.

6. Font A. Guía de tratamientos psicológicos eficaces en el cáncer. In: M. Pérez JRH, C. Fernández, I. Amigo, editor. Guía de tratamientos psicológicos eficaces II Madrid: Pirámide; 2009. p. 57-92.

7. Newell SA, Sanson-Fisher RW, Savolainen NJ. Systematic review of psychological therapies for cancer patients: Overview and recommendations for future research. J Natl Cancer Inst2002;94:55884.

8. Lepore SJ, Coyne JC. Psychological interventions for distress in cancer patients: A review of reviews. Ann Behav Med 2006;32:85-92. Doi: 10.1207/ s15324796abm3202_2.

9. Fawzy FI. Psychosocial interventions for patients with cancer: What works and what doesn't. Eur J Cancer 1999;35:155964. Doi: 10.1016/S0959-8049(99)001914.

10. Barsevick AM, Sweeney C, Haney E, Chung E. A systematic qualitative analysis of psychoeducational interventions for depression in patients with cancer. Oncol Nurs Forum 2002;29:73-84; quiz 5.

11. Cwikel JG, Behar LC. Social work with adult cancer patients: A vote-count review of intervention research. Soc Work Health Care 1999;29:39-67. Doi: 10.1300/ J010v29n02_03.

12. Devine EC, Westlake SK. The effects of psychoeducational care provided to adults with cancer: Meta-analysis of 116 studies. Oncol Nurs Forum 1995;22:1369-81.

13. Fors EA, Bertheussen GF, Thune I, Juvet LK, Elvsaas IK, Oldervoll L, et al. Psy- chosocial interventions as part of breast cancer rehabilitation programs? Results from a systematic review. Psychooncology 2011;20:909-18.

14. Hopkinson JB, Brown JC, Okamoto I, Addington-Hall JM. The effectiveness of patient-family carer (couple) intervention for the management of symptoms and other health-related problems in people affected by cancer: A systematic literature search and narrative review. J Pain Symptom Manage 2012;43:111-42. Doi: 10.1016/j. jpainsymman.2011.03.013.

15. Hoving JL, Broekhuizen MLA, FringsDresen MHW. Return to work of breast cancer survivors: A systematic review of intervention studies. BMC Cancer 2009;9. Doi: 10.1186/1471-2407-9-117.

16. Ibañez ES, J. . Intervención psicológica en enfermos de cáncer: planteamientos del presente, deseos de futuro. Análisis y Modificación de Conducta 2008;34:259-90.

17. Moyer A, Sohl SJ, Knapp-Oliver SK, Schneider S. Characteristics and methodological quality of 25 years of research investigating psychosocial interventions for cancer patients. Cancer Treat Rev 2009;35:47584. Doi: 10.1016/j.ctrv.2009.02.003.

18. Redd WH, Montgomery GH, DuHamel $\mathrm{KN}$. Behavioral intervention for cancer treatment side effects. J Natl Cancer Inst 2001;93:810-23.

19. Ross L, Boesen EH, Dalton SO, Johansen C. Mind and cancer: Does psychosocial intervention improve survival and psychological well-being? Eur J Cancer 2002;38:1447-57. Doi: 10.1016/S09598049(02)00126-0.

20. Steel JL, Bress K, Popichak L, Evans JS, Savkova A, Biala $M$, et al. A systematic review of randomized controlled trials testing the efficacy of psychosocial interventions for gastrointestinal cancers. J Gastrointest Cancer 2014;45:181-9. Doi: 10.1007/s12029-014-9605-z.

21. Dura E, Ibanez E. The psychosocial effects of an information program involving Spa- 
nish breast cancer patients. J Psychosoc Oncol 1991;9:45-65.

22. Fogarty LA, Curbow BA, Wingard JR, McDonnell K, Somerfield MR. Can 40 seconds of compassion reduce patient anxiety. J Clin Oncol 1999;17:371-9.

23. Jensen AB, Madsen B, Andersen P, Rose C. Information for cancer patients entering a clinical trial-an evaluation of an information strategy. Eur J Cancer 1993;29:2235-8. Doi: 10.1016/0959-8049(93)90213-Y.

24. McQuellon RP, Wells M, Hoffman S, Craven B, Russell G, Cruz J, et al. Reducing distress in cancer patients with an orientation program. Psychooncology 1998;7:207-17. Doi: 10.1002/(SICI)10991611 (199805/06) 7:3<207::AID PON304>3.0.CO;2-T.

25. Ream E, Richardson A. The role of information in patients $\square$ adaptation to chemotherapy and radiotherapy: A review of the literature. Eur J Cancer Care 1996;5:132-8.

26. Yu HL, Li Y, Mao XQ, Ma R, Sun JZ, Pan F. Physiological and psychological improvements of Chinese women with breast cancer in perioperative period after brief structured psychotherapy. Chin Med 2007;120:74-6.

27. Lerman R, Jarski R, Rea H, Gellish R, Vicini $F$. Improving symptoms and quality of life of female cancer survivors: A randomized controlled study. Ann Surg Oncol 2012;19:373-8. Doi: 10.1245/s10434011-2051-2.

28. Sandgren AK, McCaul KD. Long-term telephone therapy outcomes for breast cancer patients. Psychooncology 2007;16:38-47. Doi: 10.1002/pon.1038.

29. Gruber BL, Hersh SP, Hall NRS, Waletzky LR, Kunz JF, Carpenter JK, et al. Immunological responses of breast cancer patients to behavioral interventions. Biofeedback Self Regul 1993;18:1-22. Doi: 10.1007/ BF00999510.

30. Greer S, Moorey S, Baruch JDR, Watson M, Robertson BM, Mason A, et al. Adjuvant psychological therapy for patients with cancer: A prospective randomised trial. BMJ 1992;304:675-80.

31. Coyne JC, Lepore SJ, Palmer SC. Efficacy of psychosocial interventions in cancer care: Evidence is weaker than it first looks. Ann Behav Med 2006;32:104-10. Doi: 10.1207/s15324796abm3202_5.

32. Fawzy Fl, Fawzy NW, Arndt LA, Pasnau RO. Critical review of psychosocial interventions in cancer care. Arch Gen Psychiatry 1995;52:100-13.

33. Hosaka T, Sugiyama Y, Hirai K, Okuyama T, Sugawara Y, Nakamura Y. Effects of a modified group intervention with earlystage breast cancer patients. General Hospital Psychiatry 2001;23:145-51. Doi: 10.1016/S0163-8343(01)00131-1.

34. Kerr J, Engel J, Schlesinger-Raab A, Sauer $H$, Hölzel D. Communication, quality of life and age: Results of a 5-year prospective study in breast cancer patients. Ann Oncol 2003;14:421-7. Doi: 10.1093/annonc/mdg098.

35. Burton MV, Parker RW, Farrell A, Bailey D, Conneely J, Booth $\mathrm{S}$, et al. A randomized controlled trial of preoperative psychological preparation for mastectomy. Psychooncology 1995;4:1-19. Doi: 10.1002/ pon.2960040102.

36. Edgar L, Rosberger Z, Nowlis D. Coping with cancer during the first year after diagnosis: Assessment and intervention. Cancer 1992;69:817-28.

37. Pruitt B, Waligora-Serafin, B., McMahon, T., Byrd, G., Besselman, L., Kelly, G., et al. An educational intervention for newlydiagnosed cancer patients undergoing radiotherapy. Psychooncology 1993;2:5562. Doi: 10.1002/pon.2960020108.

38. Chen E, Zeltzer LK, Craske MG, Katz ER. Alteration of memory in the reduction of children's distress during repeated aversive medical procedures. J Consult Clin Psychol 1999;67:481-90. Doi: 10.1037/0022006X.67.4.481.

39. Syrjala KL, Donaldson GW, Davis MW, Kippes ME, Carr JE. Relaxation and ima- 
gery and cognitive-behavioral training reduce pain during cancer treatment: A controlled clinical trial. Pain 1995;63:189-98. Doi: 10.1016/0304-3959(95)00039-U.

40. Syrjala KL, Cummings C, Donaldson GW. Hypnosis or cognitive behavioral training for the reduction of pain and nausea during cancer treatment: A controlled clinical trial. Pain 1992;48:137-46. Doi: 10.1016/0304-3959(92)90049-H.

41. Pellicer X. Applications of hypnosis in oncology. Psiquis 1994;15:51-9.

42. Redd WH, Andresen GV, Minagawa RY. Hypnotic control of anticipatory emesis in patients receiving cancer chemotherapy. J Consult Clin Psychol 1982;50:14-9. Doi: 10.1037//0022-006X.50.1.14.

43. Redd $\mathbf{W H}$, Jacobsen PB, Die-Trill M, Dermatis $\mathrm{H}$, McEvoy M, Holland JC. Cognitive/attentional distraction in the control of conditioned nausea in pediatric cancer patients receiving chemotherapy. J Consult Clin Psychol 1987;55:391-5.

44. John LD. Self-care strategies used by patients with lung cancer to promote quality of life. Oncol Nurs Forum 2010;37:33947. Doi: 10.1188/10.ONF.339-347.

45. Carayol M, Delpierre C, Bernard P, Ninot G. Population-, intervention- and methodology-related characteristics of clinical trials impact exercise efficacy during adjuvant therapy for breast cancer: A meta-regression analysis. Psychooncology 2015;24:737-47. Doi: 10.1002/pon.3727.

46. Rapkin DA, Straubing M, Holroyd JC. Guided imagery, hypnosis and recovery from head and neck cancer surgery: An exploratory study. International J Clin Exp Hyp 1991;39:215-26.

47. Badger TA, Braden CJ, Mishel MH. Depression burden, self-help interventions, and side effect experience in women receiving treatment for breast cancer. Oncol Nurs Forum 2001;28:567-74.

48. Alter CL, Fleishman SB, Kornblith AB, HoIland JC, Biano D, Levenson R, et al. Supportive telephone intervention for patients receiving chemotherapy: A pilot study. Psychosomatics 1996;37:425-31.

49. Maguire P, Brooke M, Tait A, Thomas C, Sellwood R. The effect of counselling on physical disability and social recovery after mastectomy. Clin Oncol 1983;9:319-24.

50. McLachlan SA, Allenby A, Matthews J, Wirth A, Kissane D, Bishop $M$, et al. Randomized trial of coordinated psychosocial interventions based on patient self-assessments versus standard care to improve the psychosocial functioning of patients with cancer. J Clinl Oncol 2001;19:4117-25.

51. Jahn P, Kuss O, Schmidt H, Bauer A, Kitzmantel $M$, Jordan $K$, et al. Improvement of pain-related self-management for cancer patients through a modular transitional nursing intervention: A cluster-randomized multicenter trial. Pain 2014;155:746-54. Doi: 10.1016/j.pain.2014.01.006.

52. Duijts SFA, Faber MM, Oldenburg HSA, Van Beurden M, Aaronson NK. Effectiveness of behavioral techniques and physical exercise on psychosocial functioning and health-related quality of life in breast cancer patients and survivors-a meta-analysis. Psychooncology 2011;20:115-26. Doi: 10.1002/pon.1728.

53. Penttinen HM, Saarto T, Kellokumpu-Lehtinen P, Blomqvist C, Huovinen R, Kautiainen $\mathrm{H}$, et al. Quality of life and physical performance and activity of breast cancer patients after adjuvant treatments. Psychooncology 2011;20:1211-20. Doi: 10.1002/pon.1837.

54. Schmitz K. Physical activity and breast cancer survivorship. Recent Results Cancer Res 186:189-215. Doi: 10.1007/9783-642-04231-7

55. Rogers LQ, Markwell SJ, Courneya KS, McAuley E, Verhulst S. Physical activity type and intensity among rural breast cancer survivors: Patterns and associations with fatigue and depressive symptoms. J Cancer Surviv 2011;5:54-61. Doi: 10.1007/s11764-010-0160-8. 
56. Ochoa C, Sumalla EC, Maté J, Castejón V, Rodríguez A, Blanco I, et al. Positive group psychotherapy in cancer. Psicooncologia. 2010;7:7-34.

57. Thompson J, Coleman R, Colwell B, Freeman J, Greenfield D, Holmes K, et al. Preparing breast cancer patients for survivorship (PREP) - A pilot study of a patientcentred supportive group visit intervention. Eur J Oncol Nurs 2014;18:10-6. Doi: 10.1016/j.ejon.2013.10.004.

58. Fillion L, Gagnon P, Leblond F, Gélinas C, Savard J, Dupuis R, et al. A brief intervention for fatigue management in breast cancer survivors. Cancer Nurs 2008;31:145-59. Doi: 10.1097/01. NCC.0000305698.97625.95.

59. Ridner SH, Dietrich MS, Kidd N. Breast cancer treatment-related lymphedema self-care: Education, practices, symptoms, and quality of life. Support Care Cancer 2011;19:63137. Doi: 10.1007/s00520-010-0870-5.

60. Spiegel D, Kraemer H, Bloom J, Gottheil E. Effect of psychosocial treatment on survival of patients with metastatic breast cancer. Lancet 1989;334:888-91. Doi: 10.1016/S0140-6736(89)91551-1.

61. Spiegel D, Butler LD, Giese-Davis J, Koopman C, Miller E, DiMiceli S, et al. Effects of supportive-expressive group therapy on survival of patients with metastatic breast cancer: A randomized prospective trial. Cancer 2007;110:1130-8. Doi: 10.1002/ cncr.22890.

62. Andersen $\mathrm{BL}$, Thornton $\mathrm{LM}$, Shapiro $\mathrm{CL}$, Farrar WB, Mundy BL, Yang $\mathrm{HC}$, et al. Biobehavioral, immune, and health benefits following recurrence for psychological intervention participants. Clin Cancer Res 2010;16:3270-8. Doi: 10.1158/10780432.CCR-10-0278.

63. Andersen BL, Yang HC, Farrar WB, GoldenKreutz DM, Emery CF, Thornton LM, et al. Psychologic intervention improves survival for breast cancer patients: A randomized clinical trial. Cancer 2008;113:34508. Doi: 10.1002/cncr.23969.
64. Daniels J, Kissane DW. Psychosocial interventions for cancer patients. Curr Opin Oncol 2008;20:367-71. Doi: 10.1097/ CCO.0b013e3283021658.

65. Edelman S, Lemon J, Bell DR, Kidman AD. Effects of group CBT on the survival time of patients with metastatic breast cancer. Psychooncology. 1999;8:474-81. Doi: 10.1002/(SICI)10991611 ( $199911 / 12) 8: 6<474:$ :AID PON427>3.0.CO;2-A.

66. Fawzy Fl, Fawzy NW, Hyun CS, Elashoff R, Guthrie D, Fahey JL, et al. Malignant melanoma: Effects of an early structured psychiatric intervention, coping, and affective state on recurrence and survival 6 years later. Arch GenPsychiatry 1993;50:681-9.

67. Gellert GA, Maxwell RM, Siegel BS. Survival of breast cancer patients receiving adjunctive psychosocial support therapy: A 10-year follow-up study. J Clin Oncol 1993;11:66-9.

68. Kissane D, Li Y. Effects of supportiveexpressive group therapy on survival of patients with metastatic breast cancer: A randomized prospective trial [1]. Cancer 2008;112:443-4. Doi: 10.1002/ cncr.23179.

69. Kissane DW, Love A, Hatton A, Bloch S, Smith G, Clarke DM, et al. Effect of cognitive-existential group therapy on survival in early-stage breast cancer. J Clin Oncol 2004;22:4255-60. Doi: 10.1200/ JCO.2004.12.129.

70. Linn MW, Linn BS, Harris R. Effects of counseling for late stage cancer patients. Cancer 1982;49:1048-55.

71. Morgenstern H, Gellert GA, Walter SD, Ostfeld AM, Siegel BS. The impact of a psychosocial support program on survival with breast cancer: The importance of selection bias in program evaluation. J Chronic Dis 1984;37:273-82. Doi: 10.1016/0021-9681(84)90135-8.

72. Spiegel D, Morrow GR, Classen C, Raubertas R, Stott PB, Mudaliar N, et al. Group psychotherapy for recently diag- 
nosed breast cancer patients: A multicenter feasibility study. Psychooncology 1999;8:482-93. Doi: 10.1002/(SICI)10991611 (199911/12)8:6<482::AID PON402>3.0.CO;2-W.

73. Reich RR, Lengacher CA, Kip KE, Shivers SC, Schell MJ, Shelton MM, et al. Baseline Immune Biomarkers as Predictors of MBSR(BC) Treatment Success in Off-Treatment Breast Cancer Patients. Biol Res Nurs 2014;16:429-37. Doi: 10.1177/1099800413519494.

74. Tong G, Geng Q, Cheng J, Chai J, Xia Y, Feng $R$, et al. Effects of psycho-behavioral interventions on immune functioning in cancer patients: A systematic review. J Cancer Res Clin Oncol 2014;140:15-33. Doi: 10.1007/s00432-013-1516-z.

75. Allison H, Gripton J, Rodway M. Social work services as a component of palliative care with terminal cancer patients. Soc Work Health Care 1983;8:29-44.

76. Connor SR. Denial in terminal illness: to intervene or not to intervene. The Hospice J 1992;8:1-15.

77. Syrjala KL, Abrams JR, Polissar NL, Hansberry J, Robison J, DuPen S, et al. Patient training in cancer pain management using integrated print and video materials: A multisite randomized controlled trial. Pain 2008;135:175-86. Doi: 10.1016/j. pain.2007.10.026.

78. Armes J, Chalder T, Addington-Hall J, Richardson A, Hotopf M. A randomized controlled trial to evaluate the effectiveness of a brief, behaviorally oriented intervention for cancer-related fatigue. Cancer 2007;110:1385-95. Doi: 10.1002/ cncr.22923.

79. Given C, Given B, Rahbar M, Jeon S, McCorkle R, Cimprich B, et al. Effect of a cognitive behavioral intervention on reducing symptom severity during chemotherapy. J Clinl Oncol 2004;22:507-16. Doi: 10.1200/JCO.2004.01.241.

80. Salonen P, Tarkka MT, Kellokumpu-Lehtinen PL, Åstedt-Kurki P, Luukkaala T,
Kaunonen M. Telephone intervention and quality of life in patients with breast cancer. Cancer Nurs. 2009;32:177-90. Doi: 10.1097/NCC.0b013e31819b5b65.

81. Chujo M, Mikami I, Takashima S, Saeki T, Ohsumi S, Aogi K, et al. A feasibility study of psychosocial group intervention for breast cancer patients with first recurrence. Support Care Cancer 2005;13:503-14. Doi: 10.1007/s00520-004-0733-z.

82. Classen C, Butler LD, Koopman C, Miller E, DiMiceli S, Giese-Davis J, et al. Supportive-expressive group therapy and distress in patients with metastatic breast cancer: A randomized clinical intervention trial. Arch Gen Psychiatry 2001;58:494-501.

83. Winzelberg AJ, Classen C, Alpers GW, Roberts H, Koopman C, Adams RE, et al. Evaluation of an internet support group for women with primary breast cancer. Cancer 2003;97:1164-73. Doi: 10.1002/ cncr. 11174.

84. Lapid MI, Rummans TA, Brown PD, Frost $M H$, Johnson ME, Huschka MM, et al. Improving the quality of life of geriatric cancer patients with a structured multidisciplinary intervention: A randomized controIled trial. Palliat Support Care 2007;5:10714. Doi: 10.1017/S1478951507070174.

85. Griffiths J, Ewing G, Rogers M. «Moving swiftly on.» psychological support provided by district nurses to patients with palliative care needs. Cancer Nurs 2010;33:390-7. Doi: 10.1097/NCC.0b013e3181d55f9b.

86. Hunt RW, Bond MJ, Pater GD. Psychological responses to cancer: A case for cancer support groups. Community Health Stud 1990;14:35-8.

87. Chambers SK, Ferguson M, Gardiner RA, Aitken J, Occhipinti S. Intervening to improve psychological outcomes for men with prostate cancer. Psychooncology 2013;22:1025-34. Doi: 10.1002/pon.3095.

88. Capozzo MA, Martinis E, Pellis G, Giraldi T. An early structured psychoeducational intervention in patients with breast cancer: Results from a feasibility study. Can- 
cer Nurs 2010;33:228-34. Doi: 10.1097/ NCC.0b013e3181c1acd6.

89. Lieberman MA, Golant M, Giese-Davis J, Winzlenberg A, Benjamin $H$, Humphreys K, et al. Electronic support groups for breast carcinoma: A clinical trial of effectiveness. Cancer 2003;97:920-5. Doi: 10.1002/cncr.11145.

90. Fish JA, Ettridge K, Sharplin GR, Hancock B, Knott VE. Mindfulness-based Cancer Stress Management: Impact of a mindfulnessbased programme on psychological distress and quality of life. Eur J Cancer Care 2014;23:413-21. Doi: 10.1111/ecc.12136.

91. Kvillemo P, Bränström R. Experiences of a mindfulness-based stress-reduction intervention among patients with cancer. Cancer Nurs 2011;34:24-31. Doi: 10.1097/ NCC.0b013e3181e2d0df.

92. Velikova G, Booth L, Smith AB, Brown PM, Lynch P, Brown JM, et al. Measuring quality of life in routine oncology practice improves communication and patient well-being: A randomized controlled trial. J Clin Oncol 2004;22:714-24. Doi: 10.1200/JCO.2004.06.078.

93. Vargas $\mathrm{S}$, Antoni $\mathrm{MH}$, Carver CS, Lechner SC, Wohlgemuth W, Llabre M, et al. Sleep quality and fatigue after a stress management intervention for women with earlystage breast cancer in Southern Florida. Int J Behav Med 2014;21:971-81. Doi: 10.1007/s12529-013-9374-2.

94. Sandgren AK, McCaul KD, King B, O)Donnell S, Foreman G. Telephone therapy for patients with breast cancer. Oncol Nurs Forum 2000;27:683-8.

95. Lee JY, Park HY, Jung D, Moon M, Keam B, Hahm BJ. Effect of brief psychoeducation using a tablet PC on distress and quality of life in cancer patients undergoing chemotherapy: A pilot study. Psychooncology 2014;23:928-35. Doi: 10.1002/pon.3503.

96. O>Connor G, Coates V, O>Neill S. Randomised controlled trial of a tailored information pack for patients undergoing surgery and treatment for rectal cancer.
Eur J Oncol Nurs 2014;18:183-91. Doi: 10.1016/j.ejon.2013.10.011.

97. Borneman T, Koczywas M, Sun VCY, Piper BF, Uman G, Ferrell B. Reducing patient barriers to pain and fatigue management. J Pain Symptom Manage 2010;39:486-501. Doi: 10.1016/j.jpainsymman.2009.08.007.

98. Lin CC, Chou PL, Wu SL, Chang YC, Lai YL. Long-term effectiveness of a patient and family pain education program on overcoming barriers to management of cancer pain. Pain 2006;122:271-81. Doi: 10.1016/j.pain.2006.01.039.

99. Miaskowski C, Dodd M, West C, Schumacher K, Paul SM, Tripathy D, et al. Randomized clinical trial of the effectiveness of a self-care intervention to improve cancer pain management. J Clinl Oncol 2004;22:1713-20. Doi: 10.1200/ JCO.2004.06.140.

100. Wells N, Hepworth JT, Murphy BA, Wujcik D, Johnson R. Improving cancer pain management through patient and family education. J Pain Symptom Manage 2003;25:344-56. Doi: 10.1016/S08853924(02)00685-1.

101. Ward S, Donovan HS, Owen B, Grosen E, Serlin R. An individualized intervention to overcome patient-related barriers to pain management in women with gynecologic cancers. Res Nurs Health 2000;23:393405.

102. Clotfelter CE. The effect of an educational intervention on decreasing pain intensity in elderly people with cancer. Oncol Nurs Forum 1999;26:27-33.

103. De Wit R, Van Dam F, Zandbelt L, Van Buuren A, Van Der Heijden K, Leenhouts $\mathrm{G}$, et al. A Pain education program for chronic cancer pain patients: Follow-up results from a randomized controlled trial. Pain 1997;73:55-69. Doi: 10.1016/S03043959(97)00070-5.

104. Zhang M, Chan SWC, You L, Wen Y, Peng $L$, Liu $W$, et al. The effectiveness of a selfefficacy-enhancing intervention for Chi- 
nese patients with colorectal cancer: A randomized controlled trial with 6-month follow up. Intl J Nurs Stud 2014;51:108392. Doi: 10.1016/j.ijnurstu.2013.12.005.

105. Groarke A, Curtis R, Kerin M. Cognitivebehavioural stress management enhances adjustment in women with breast cancer. Brit J Health Psych 2013;18:623-41. Doi: 10.1111/bjhp.12009.

106. Dolbeault S, Cayrou S, Brédart A, Viala $A L$, Desclaux B, Saltel P, et al. The effectiveness of a psycho-educational group after early-stage breast cancer treatment: Results of a randomized French study. Psychooncology 2009;18:647-56. Doi: 10.1002/pon.1440.

107. Kissane DW, Bloch S, Smith GC, Miach P, Clarke DM, Ikin J, et al. Cognitiveexistential group psychotherapy for women with primary breast cancer: A randomized controlled trial. Psychooncology 2003;12:532-46. Doi: 10.1002/pon.683.

108. Van Der Meulen IC, May AM, Ros WJG, Oosterom M, Hordijk GJ, Koole R, et al. One-year effect of a nurse-led psychosocial intervention on depressive symptoms in patients with head and neck cancer: A randomized controlled trial. Oncologist 2013;18:336-44. Doi: 10.1634/theoncologist.2012-0299.

109. Würtzen $H$, Dalton $S O$, Elsass $P$, Sumbundu AD, Steding-Jensen M, Karlsen RV, et al. Mindfulness significantly reduces self-reported levels of anxiety and depression: Results of a randomised controlled trial among 336 Danish women treated for stage I-III breast cancer. Eur J Cancer 2013;49:13651373. Doi: 10.1016/j.ejca.2012.10.030.

110. Bränström R, Kvillemo $P$, Moskowitz JT. A randomized study of the effects of mindfulness training on psychological wellbeing and symptoms of stress in patients treated for cancer at 6-month follow-up. Inter J Behav Med 2012;19:535-42. Doi: 10.1007/s12529-011-9192-3.

111. David N, Schlenker P, Prudlo U, Larbig W. Internet-based program for coping with cancer: A randomized controlled trial with hematologic cancer patients. Psychooncology 2013;22:1064-72. Doi: 10.1002/ pon.3104.

112. Garssen B, Boomsma MF, De Jager Meezenbroek E, Porsild T, Berkhof J, Berbee M, et al. Stress management training for breast cancer surgery patients. Psychooncology 2013;22:572-80. Doi: 10.1002/pon.3034.

113. Gaston-Johansson F, Fall-Dickson JM, Nanda JP, Sarenmalm EK, Browall M, Goldstein N. Long-term effect of the self-management comprehensive coping strategy program on quality of life in patients with breast cancer treated with high-dose chemotherapy. Psychooncology 2013;22:5309. Doi: 10.1002/pon.3031.

114. Espinoza M, Baños RM, García-Palacios A, Cervera JM, Esquerdo G, Barrajón E, et al. Promotion of emotional wellbeing in oncology inpatients using VR. Stud Health Tech Inform 2012;181:53-7. Doi: 10.3233/978-1-61499-121-2-53.

115. Phillips KM, Jim HSL, Small BJ, Tanvetyanon T, Roberts WS, Jacobsen PB. Effects of self-directed stress management training and home-based exercise on stress management skills in cancer patients receiving chemotherapy. Stress Health 2012;28:36875. Doi: 10.1002/smi.2450.

116. Maryam A, Fazlollah A, Eesa M, Ebrahim $H$, Abbas $V$. The effect of designed exercise programme on quality of life in women with breast cancer receiving chemotherapy. Scand J Caring Sci 2010;24:251-8. Doi: 10.1111/j.1471-6712.2009.00714.x.

117. Rogers LQ, Hopkins-Price P, Vicari S, Markwell S, Pamenter R, Courneya KS, et al. Physical activity and health outcomes three months after completing a physical activity behavior change intervention: Persistent and delayed effects. Cancer Epidemiol Biomarkers Prev 2009;18:1410-8. Doi: 10.1158/1055-9965.EPI-08-1045.

118. Mutrie N, Campbell AM, Whyte F, McConnachie A, Emslie C, Lee L, et al. Benefits of supervised group exercise program- 
me for women being treated for early stage breast cancer: Pragmatic randomised controlled trial. BMJ 2007;334:517-20. Doi: 10.1136/bmj.39094.648553.AE.

119. Mock V, Frangakis C, Davidson NE, Ropka ME, Pickett M, Poniatowski B, et al. Exercise manages fatigue during breast cancer treatment: A randomized controlled trial. Psychooncology 2005;14:464-77. Doi: 10.1002/pon.863.

120. Trask PC, Paterson AG, Griffith KA, Riba MB, Schwartz JL. Cognitive-behavioral intervention for distress in patients with melanoma: Comparison with standard medical care and impact on quality of life. Cancer 2003;98:854-64. Doi: 10.1002/ cncr.11579.

121. Given B, Given CW, McCorkle R, Kozachik S, Cimprich B, Rahbar MH, et al. Pain and fatigue management: results of a nursing randomized clinical trial. Oncol Nurs Forum 2002;29:949-56.

122. Iruarrizaga, I. Psicobiología, neuropsicología y desórdenes emocionales. Intervención cognitivo-conductual en el tratamiento de las naúseas y vómitos asociados a la quimioterapia en pacientes con cáncer de mama. I Congreso Virtual de Psiquiatría 1 de Febrero - 15 de Marzo 2000; Conferencia 58-Cl-I: [17 pantallas]. Disponible en: http://www.psiquiatria.com/congreso/ mesas/mesa58/conferencias/58_ci_i.htm

123. Park JH, Bae SH, Jung YS, Kim KS. Quality of life and symptom experience in breast cancer survivors after participating in a psychoeducational support program: A pilot study. Cancer Nurs 2012;35:E34-E41. Doi: 10.1097/NCC.0b013e318218266a.

124. Meneses KD, McNees P, Loerzel VW, Su $X$, Zhang $Y$, Hassey LA. Transition from treatment to survivorship: Effects of a psychoeducational intervention on quality of life in breast cancer survivors. Oncol Nurs Forum 2007;34:1007-16. Doi: 10.1188/07.ONF.1007-1016.

125. Work and cancer: How cancer affects working lives. CancerBACUP Ashford Co- lour Press. [Internet]. [Acceso 15 de octubre de 2015] Disponible en: http: //www. cancerbacup.org.uk/News/Press/Pressreleasesstaments/2005/45691514 2008

126. Verbeek J, Spelten E, Kammeijer M, Sprangers M. Return to work of cancer survivors: A prospective cohort study into the quality of rehabilitation by occupational physicians. Occup Environ Med 2003;60:352-7. Doi: 10.1136/ oem.60.5.352.

127. Hoffman CJ, Ersser SJ, Hopkinson JB, Nicholls PG, Harrington JE, Thomas PW. Effectiveness of mindfulness-based stress reduction in mood, breast- and endocrine-related quality of life, and well-being in stage 0 to III breast cancer: A randomized, controlled trial. J Clin Oncol 2012;30:1335-42. Doi: 10.1200/ JCO.2010.34.0331.

128. Naumann F, Martin E, Philpott M, Smith C, Groff D, Battaglini C. Can counseling add value to an exercise intervention for improving quality of life in breast cancer survivors? A feasibility study. J Supp Oncol 2012;10:188-94. Doi: 10.1016/j. suponc.2011.09.004.

129. Lengacher CA, Johnson-Mallard V, Barta M, Fitzgerald S, Moscoso MS, Post-White J, et al. Feasibility of a mindfulness-based stress reduction program for early-stage breast cancer survivors. J Holist Nurs 2011;29:107-17.

130. Matchim Y, Armer JM, Stewart BR. Effects of mindfulness-based stress reduction (MBSR) on health among breast cancer survivors. West J Nurs Res 2011;33:9961016. Doi: 10.1177/0193945910385363.

131. Loizzo JJ, Peterson JC, Charlson ME, Wolf EJ, Altemus M, Briggs WM, et al. The effect of a contemplative self-healing program on quality of life in women with breast and gynecologic cancers. Altern Ther Health Med 2010;16:30-7.

132. Cantarero-Villanueva I, Fernández-Lao C, Cuesta-Vargas AI, Del Moral-Avila R, Fernández-De-Las-Peñas C, Arroyo-Mora- 
les $M$. The effectiveness of a deep water aquatic exercise program in cancer-related fatigue in breast cancer survivors: A randomized controlled trial. Arch Phys Med Rehabil 2013;94:221-30. Doi: 10.1016/j. apmr.2012.09.008.

133. Knobf MT, Thompson AS, Fennie K, Erdos D. The effect of a community-based exercise intervention on symptoms and quality of Life. Cancer Nurs 2014;37:E43-E50. Doi: 10.1097/NCC.0b013e318288d40e.

134. Traeger L, Penedo FJ, Benedict C, Dahn JR, Lechner SC, Schneiderman N, et al. Identifying how and for whom cognitivebehavioral stress management improves emotional well-being among recent prostate cancer survivors. Psychooncology 2013;22:250-9. Doi: 10.1002/pon.2074.

135. Choi J, Kuo CWJ, Sikorskii A, You M, Ren D, Sherwood PR, et al. Cognitive behavioral symptom management intervention in patients with cancer: Survival analysis. Supp Care Cancer 2012;20:1243-50. Doi: 10.1007/s00520-011-1210-0.

136. D'Souza V, Blouin E, Zeitouni A, Muller K, Allison PJ. An investigation of the effect of tailored information on symptoms of anxiety and depression in head and neck cancer patients. Oral Oncol 2013;49:431-7. Doi: 10.1016/j.oraloncology.2012.12.001.

137. Keefe FJ, Ahles TA, Sutton L, Dalton J, Baucom D, Pope MS, et al. Partner-guided cancer pain management at the end of life: A preliminary study. J Pain Symptom Manage 2005;29:263-72. Doi: 10.1016/j. jpainsymman.2004.06.014.

138. Bloch S, Kissane D. Psychotherapies in Psychooncology: An exciting new challenge. Br J Psychiatry 2000;177:112-6. Doi: 10.1192/bjp.177.2.112.

139. Savard J, Simard S, Giguère I, Ivers H, Morin $\mathrm{CM}$, Maunsell E, et al. Randomized clinical trial on cognitive therapy for depression in women with metastatic breast cancer: Psychological and immunological effects. Palliat Support Care 2006;4:219-37.

140. Aranda S, Schofield P, Weih L, Milne D, Yates $\mathrm{P}$, Faulkner R. Meeting the support and information needs of women with advanced breast cancer: A randomised controlled trial. Br Cancer J 2006;95:667-73. Doi: 10.1038/sj.bjc.6603320.

141. Sloman R. Relaxation and imagery for anxiety and depression control in community patients with advanced cancer. Cancer Nurs 2002;25:432-5. 